\title{
Optimal piezoelectric resistive-inductive shunt damping of plates with residual mode correction
}

\author{
Toftekær, Johan F.; Benjeddou, Ayech; Høgsberg, Jan; Krenk, Steen
}

Published in:

Journal of Intelligent Material Systems and Structures

Link to article, DOI:

$10.1177 / 1045389 \times 18798953$

Publication date:

2018

Document Version

Peer reviewed version

Link back to DTU Orbit

Citation (APA):

Toftekær, J. F., Benjeddou, A., Høgsberg, J., \& Krenk, S. (2018). Optimal piezoelectric resistive-inductive shunt damping of plates with residual mode correction. Journal of Intelligent Material Systems and Structures, 29(16), 3346-3370. https://doi.org/10.1177/1045389X18798953

\section{General rights}

Copyright and moral rights for the publications made accessible in the public portal are retained by the authors and/or other copyright owners and it is a condition of accessing publications that users recognise and abide by the legal requirements associated with these rights.

- Users may download and print one copy of any publication from the public portal for the purpose of private study or research.

- You may not further distribute the material or use it for any profit-making activity or commercial gain

- You may freely distribute the URL identifying the publication in the public portal 


\title{
Optimal piezoelectric resistive-inductive shunt damping of plates with residual mode correction
}

\author{
Johan F. Toftekær ${ }^{1}$, Ayech Benjeddou ${ }^{2,3}$, Jan Høgsberg ${ }^{1}$ and Steen Krenk ${ }^{1}$
}

\begin{abstract}
This work concerns vibration suppression of plates and plate-like structures by resonant piezoelectric damping, introduced by resistive-inductive $(R L)$ shunts. The performance of this type of shunt damping relies on the precise calibration of the shunt frequency, where an important aspect is the ability to account for the energy spill-over from the non-resonant modes, not taken into account by most available calibration methods. A newly proposed calibration procedure includes this residual mode contribution by a quasi-dynamic modal correction, taking both flexibility and inertia effects of the non-resonant modes into account. In the present work, this procedure is implemented in a finite element model combining Kirchhoff plate bending kinematics for the host structure, and a plane-stress assumption for a pair of bonded piezoceramic patches. The established model is verified by comparison with shunt calibrations from benchmark examples in the literature. As demonstrated by frequency response plots and the obtained damping ratios, the RL-shunt tuning is influenced by the effect of the non-resonant modes and omission may yield a significant detuning of the shunt circuit. Finally, an alternative method for precise evaluation of the effective (or generalized) electromechanical coupling coefficient (EMCC) is derived from the modal electromechanical equations of motion. This results in a new shunt tuning method, based on the effective EMCC obtained by the short- and open-circuit frequencies of the coupled piezo-plate structure.
\end{abstract}

\section{Keywords}

Shunt piezoelectric damping, resonant shunt calibration, quasi-dynamic residual mode correction, effective modal electromechanical coupling coefficient, plates

\section{Introduction}

This paper concerns the suppression of plate vibrations by means of resonant piezoelectric shunt damping. Piezoceramic patches, bonded to vibrating plates, have the ability to convert mechanical energy into electrical energy, that can then be dissipated in supplemental resonant shunts. The amount of converted energy is governed by the squared effective (or generalized) electromechanical coupling coefficient (EMCC), which depends on the properties of the piezoceramic patches and their placement on the host structure. Furthermore, the EMCC is a key parameter for the performance and tuning of a shunt, traditionally composed of an inductor $(L)$, calibrated such that the shunted piezoelectric device works in resonance with the host structure, and a resistor $(R)$, dissipating the converted mechanical energy into heat via the Joule effect. However, alternative passive and semi-active resonant $(R L)$ shunts have been proposed, for instance by the addition of a negative capacitance (de Marneffe and Preumont, 2008; Berardengo et al. , 2016) or by the use of switch damping (Ducarne et al. , 2010). The motivation behind most of these alternative resonant shunts is the difficulty in generating the large inductance required in shunt damping of (low-frequency) structural vibrations. The problem is commonly solved by the introduction of synthetic inductors (Thomas et al., 2012), which are active components that emulate the inductive behaviour in the desired frequency range. Nonetheless, high inductance values have recently been obtained and demonstrated for pure passive inductors with windings around a magnetic coil (Lossouarn et al. , 2017), allowing an unconditionally stable $R L$-shunt with large damping potential if a precise shunt tuning can be obtained already in the structural design phase. This can be realized using a consistent calibration procedure that explicitly takes into account the dynamic effects of the full flexible structure, as the present work shall demonstrate.

Piezoelectric vibration damping by resonant $R L$-shunts was originally suggested by Forward (1979) and further developed by Hagood and von Flotow (1991), who proposed two calibration procedures for a series shunt based on minimization of the response amplitudes and maximization of the damping characteristics. A calibration procedure for the parallel $R L$ shunt was subsequently proposed by Wu (1996), arguing that the series shunt would be inappropriate for large resistances. The series and parallel shunts have

\footnotetext{
${ }^{1}$ Department of Mechanical Engineering

Technical University of Denmark

Nils Koppels Allé, Building 404, DK-2800 Kgs. Lyngby, Denmark ${ }^{2}$ Sorbonne Universités, Université de Technologie de Compiègne, CNRS, FRE 2012 ROBERVAL

${ }^{3}$ SUPMECA, 3 Rue Fernand Hainaut, 93407 Saint Ouen, France
}

\section{Corresponding author:}

Johan F. Toftekær, Department of Mechanical Engineering, Technical University of Denmark, Nils Koppels Allé, Building 404, DK-2800 Kgs. Lyngby, Denmark

Email: jotof@mek.dtu.dk 
been compared by Park and Inman (1999), revealing a larger attainable energy dissipation using the parallel configuration, while a critical analysis of the calibration methods has been provided by Caruso (2001). Methods for multimodal piezoelectric shunt damping have been proposed by Hollkamp (1994) and Wu (1998). In later years, several alternative procedures have been proposed for the detailed tuning of $R L$-shunts, for example based on an $H_{\infty}$-norm of the response amplitude (Soltani et al, 2014) or a balanced calibration procedure based on the principle of equal modal damping (Høgsberg and Krenk , 2012), originally developed for the mechanical tuned mass damper (Krenk, 2005). The balanced calibration procedure has recently been extended to include the effects from non-resonant structural modes, first by a quasi-static correction (Krenk and Høgsberg , 2014) and then by a more general quasi-dynamic correction with both flexibility and inertia terms (Krenk and Høgsberg, 2016).

An important part of all calibration methods is the accurate determination of the squared EMCC, which governs both the attainable damping and the shunt tuning. Commonly, the squared EMCC is estimated from a single mode representation of the electromechanical structure (Thomas et al. , 2009), while improved accuracy has been demonstrated by including quasi-static effects from higher non-resonant vibration modes (Berardengo et al. , 2016). In other cases, the tuning is based directly on the squared effective EMCC determined from either experiments (Porfiri et al. , 2007; Delperro et al. , 2012) or by numerical analysis (Trindade and Benjeddou , 2009; Benjeddou , 2014). However, inherent inconsistencies between the squared modal and effective EMCC often lead to discrepancies in the tuning formulas, because the influence from non-resonant modes is neglected in the modal representation of the electromechanical structure. This motivates the present derivation of a new and consistent $R L$-shunt tuning procedure based on the effective EMCC.

The proposed shunt calibration relies on the procedure with residual mode correction, introduced by Krenk and Høgsberg (2016) for mechanical absorbers and subsequently extended to piezoelectric shunt damping in Høgsberg and Krenk (2017). It is applied to the optimal calibration of $R L$-shunted piezoceramic patches, bonded symmetrically to plate elements and structures for which the tuning is influenced by vibration modes and frequencies that are closely grouped both spectrally and spatially. It is the aim to account for the influence from the nonresonant modes on the shunt calibration, and discuss the errors associated with the pure single mode representation of the electromechanical structure, by considering both the squared modal and effective EMCC. A new modal EMCC is presented that accounts for the spill-over from non-resonant modes, whereby it becomes identical to the effective EMCC. Thus, a consistent relation is established between the modal equations of motion and the corresponding short-circuit (SC) and open-circuit (OC) frequencies of the coupled electromechanical structure. Consequently, this enables the derivation of a shunt tuning procedure based on the effective EMCC, as demonstrated in the third section of this paper.

The first section of the paper presents a finite element (FE) model for plates with a symmetrically bonded pair of piezoceramic patches in order to facilitate the subsequent analysis and assessment of the proposed $R L$-shunt tuning procedures. The plate displacements are approximated using Kirchhoff plate bending kinematics, whereas only the inplane displacements are considered for the piezoceramic patch. Equipotential conditions are imposed to all continuous patch electrodes, reducing the number of electric variables to two for the discretized piezoceramic patch. Subsequently the coupling between a pair of piezoceramic patches and a plate is established by transforming the electric forcing from the patches to the equivalent moment loads in the plate model. In the present work, a symmetric pair of identical piezoceramic patches is considered. The patches are either same-poled and parallel-wired (SP-PW) or opposite-poled and series-wired (OP-SW), whereby they operate out of phase. The number of variables in the electric domain can hereby be reduced to the voltage over the supplemental shunt by the introduction of resulting capacitance and the plane stress-reduced piezoelectric coupling coefficient for the specific patches electric connection. A thorough review of finite element techniques for the modelling of piezoelectric structural elements was provided by Benjeddou (2000).

The second section conducts a modal analysis of the SC electromechanical structure. For a specific vibration mode, the consistent modal truncation in Krenk and Høgsberg (2016) reduces the system to a single mechanical equation, in which the piezoceramic patches are represented by their resulting displacement. Hereby, the optimal tuning for both parallel and series shunt circuits (Høgsberg and Krenk, 2017) can be derived for the targeted mode of the electromechanical FE-model.

In the third section, a new shunt tuning procedure is developed based on the effective EMCC, where a simple format of the shunt tuning formulas is obtained.

In the fourth section it is demonstrated and justified that the influence from the residual modes can be directly identified from the squared modal EMCC, both with and without residual mode correction. Further, the influence on the shunt tuning when having either a SP-PW or OP-SW pair of piezoceramic patches can be seen from the new tuning formulas.

In the fifth section the established FE-model and implementation of the calibration procedures with residual mode corrections is compared with $R L$-shunt tuning results from previously proposed calibration methods for benchmark examples with a cantilever beam (Thomas et al. , 2009) and a simply supported plate (Gardonio and Casagrande, 2017). For the cantilever, available experimental data are used for comparison with the proposed FE based tuning methods. Both examples analyze the ability to achieve optimal damping by the proposed calibration procedures and validate the equality between the squared effective EMCC, and the squared modal EMCC with residual mode correction.

As a closure of this introduction, it is worth emphasising the originality of the present work with regards of the related earlier publications Høgsberg and Krenk (2012, 2015, 2017). In previous works, the balanced shunt tuning has been considered with and without residual mode correction for beam structures. Thus, the present implementation for plates and two-dimensional piezoceramic patches is a novel extension that relies on the derived FE-model. Furthermore, optimal tuning formulas have here been derived directly 
from the underlying electric equations. Besides, a new methodology for the precise evaluation of the effective EMCC is proposed. It is based on the corresponding modal EMCC with residual mode correction. Following the accurate evaluation of the effective EMCC, an alternative tuning procedure based on the latter is proposed in the third section which, as well, is a new contribution. Finally, the present shunt tuning procedures with residual mode corrections are assessed by comparison with benchmark examples for beam and plate structures.

\section{Electromechanical Piezo-Plate Model}

This first section is devoted to the derivation of a FE-model for the coupled piezo-plate structure. Initially, the variational formulations are obtained for the host plate structure and the piezoceramic patch separately, by considering the respective kinematic and constitutive relations. Subsequently, the coupling is established by transforming the electric forcing from a considered pair of piezoceramic patches to the equivalent moment loads in the plate model. Alternatively, a layer model could have been established by the use of sandwich theory (Benjeddou, 2002).

\section{Host elastic plate}

The host elastic structure is considered to cover the three-dimensional (3D) domain $\Omega$, subjected to prescribed displacements $\bar{u}_{i}$ on part of the boundary $\Gamma_{u}$, and prescribed surface tractions $F_{i}$ on the remaining part of the domain boundary $\Gamma_{F}$, such that $\partial \Omega=\Gamma_{u} \cup \Gamma_{F}$ and $\Gamma_{u} \cap \Gamma_{F}=\emptyset$. Hereby, the dynamic equilibrium, under free-body loads, and boundary equations, can be written as

$$
\begin{aligned}
\sigma_{i j, j} & =\rho \ddot{u}_{i} & & \text { in } \Omega \\
\sigma_{i j} n_{j} & =F_{i} & & \text { on } \Gamma_{F} \\
u_{i} & =\bar{u}_{i} & & \text { on } \Gamma_{u}
\end{aligned}
$$

where $i, j$ denote the 3D vector and tensor components, with repeated subscripts implying summation. Partial differentiation with respect to a space coordinate is denoted by comma, and time differentiation by a dot. The material density of the plate is denoted $\rho$, and $n_{j}$ are the components of the outward unit normal to $\Omega$.

The variational formulation is established using the weighted residual method by multiplying the equation of motion (1) with the virtual displacement $\delta u_{i}$, followed by an integration over the structural domain,

$$
\int_{\Omega} \delta u_{i}\left(\sigma_{i j, j}-\rho \ddot{u}_{i}\right) d \Omega=0
$$

Integration by parts then, using the kinematically admissible boundary conditions, gives the weak variational formulation

$$
\int_{\Omega} \delta \varepsilon_{i j} \sigma_{i j} d \Omega+\int_{\Omega} \delta u_{i} \rho \ddot{u}_{i} d \Omega=\int_{\Gamma_{F}} \delta u_{i} F_{i} d \Gamma
$$

where the displacement has to satisfy the essential boundary condition (3).

In the present work, plates and plate-like structures with thickness $t_{0}$ significantly smaller than the in-plane dimensions of the surface area $A$ are considered. Thus, a good representation of the structural behaviour is obtained by Kirchhoff plate theory. The latter considers a plane stress state, whereby the constitutive relation between the strains and stresses is reduced to the relation between the in-plane components. For an isotropic material

$$
\boldsymbol{\sigma}=\frac{E}{1-\nu} \mathbf{D}_{p} \varepsilon
$$

with Young's modulus $E$, Poisson's ratio $\nu$, and the stress and strain vectors and constitutive matrix defined as

$$
\boldsymbol{\sigma}=\left[\begin{array}{c}
\sigma_{x x} \\
\sigma_{y y} \\
\sigma_{x y}
\end{array}\right], \quad \boldsymbol{\varepsilon}=\left[\begin{array}{c}
\varepsilon_{x x} \\
\varepsilon_{y y} \\
2 \varepsilon_{x y}
\end{array}\right], \quad \mathbf{D}_{p}=\frac{1}{1+\nu}\left[\begin{array}{ccc}
1 & \nu & 0 \\
\nu & 1 & 0 \\
0 & 0 & \frac{1-\nu}{2}
\end{array}\right]
$$

The particular form of $\mathbf{D}_{p}$ becomes convenient in the subsequent derivations. Considering bending only, the inplane strains are determined from the transverse coordinate $z$ and vertical displacement $w$ as

$$
\varepsilon=z \kappa=-z \Delta w
$$

where $\Delta$ is a two-dimensional Laplace-like operator, defined as

$$
\boldsymbol{\Delta}=\boldsymbol{\nabla}_{p} \boldsymbol{\nabla}=\left[\begin{array}{cc}
\frac{\partial}{\partial x} & 0 \\
0 & \frac{\partial}{\partial y} \\
\frac{\partial}{\partial y} & \frac{\partial}{\partial x}
\end{array}\right]\left[\begin{array}{c}
\frac{\partial}{\partial x} \\
\frac{\partial}{\partial y}
\end{array}\right]=\left[\begin{array}{c}
\frac{\partial^{2}}{\partial x^{2}} \\
\frac{\partial^{2}}{\partial y^{2}} \\
2 \frac{\partial^{2}}{\partial x \partial y}
\end{array}\right]
$$

Using the constitutive relation (6), the strain definition (8), and the Laplace-like operator (9), the variational formulation (5) can be written as

$$
\int_{A} \delta \mathrm{w}\left(\boldsymbol{\Delta}^{T} \mathbf{D} \boldsymbol{\Delta}-\omega^{2} \rho t_{0}\right) \mathrm{w} d A=\int_{A_{F}} \delta \mathrm{w} F_{w} d A
$$

where harmonic solutions $w=\mathrm{w} \exp (i \omega t)$, of circular frequency $\omega$, are assumed, and the boundary forces are given only by the vertical surface traction $F_{w}$ on $\Gamma_{F}=A_{F}$. In (10), the constitutive matrix for the isotropic plate is

$$
\mathbf{D}=\frac{E t_{0}^{3}}{12(1-\nu)} \mathbf{D}_{p}
$$

The FE-formulation is now obtained from the variational formulation (10) by discretizing the amplitude of the displacement field as

$$
\mathrm{w}(x, y)=\mathbf{N}(x, y) \mathbf{u}^{e}
$$

where the shape functions in $\mathbf{N}$ and nodal degrees of freedom (dofs) in the harmonic amplitude vector $\mathbf{u}^{e}$ for element $e$ are

$$
\begin{aligned}
\mathbf{N} & =\left[\begin{array}{lllllll}
N_{w}^{1} & N_{\phi}^{1} & N_{\psi}^{1} & \cdots & N_{w}^{4} & N_{\phi}^{4} & N_{\psi}^{4}
\end{array}\right] \\
\mathbf{u}^{e} & =\left[\begin{array}{llllllll}
w_{1} & \phi_{1} & \psi_{1} & \cdots & w_{4} & \phi_{4} & \psi_{4}
\end{array}\right]^{T}
\end{aligned}
$$

The numerical index $1, \ldots, 4$ denotes the node number in the considered rectangular plate element. The shape functions $N_{w}^{i}, N_{\phi}^{i}$ and $N_{\psi}^{i}$ are derived for the nodal vertical displacement $w_{i}$ and the associated nodal rotations $\phi_{i}$ and $\psi_{i}$ about the $x$ - and $y$-axis, respectively, corresponding to a cubic Hermitian interpolation of the vertical displacement field. 
By substitution of (12) into the variational equation (10), followed by summation over the number of elements $n$, the equation of motion for the discretized plate can be written as

$$
\sum_{e=1}^{n}\left(\delta \mathbf{u}^{e}\right)^{T}\left\{\left(\mathbf{K}_{0}^{e}-\omega^{2} \mathbf{M}_{0}^{e}\right) \mathbf{u}^{e}-\mathbf{f}^{e}\right\}=0
$$

where the element mass and stiffness matrices have been introduced as

$$
\begin{aligned}
& \mathbf{M}_{0}^{e}=\int_{A^{e}} \mathbf{N}^{T} \rho t_{0} \mathbf{N} d A^{e}, \\
& \mathbf{K}_{0}^{e}=\int_{A^{e}}(\boldsymbol{\Delta} \mathbf{N})^{T} \mathbf{D}(\boldsymbol{\Delta} \mathbf{N}) d A^{e}
\end{aligned}
$$

and the external element force vector

$$
\mathbf{f}^{e}=\int_{A_{F}^{e}} \mathbf{N}^{T} F_{w} d A^{e}
$$

It is noted that four point Gauss integration is sufficient to integrate the highest polynomial order exactly.

The global mass and stiffness matrices $\mathbf{M}_{0}$ and $\mathbf{K}_{0}$, and the force $\mathbf{f}$ and displacement $\mathbf{u}$ vectors are then assembled using a standard FEM assembly procedure, adding each mass, stiffness and force component to a defined global nodal order, whereby the full discretized equation of motion can be written as

$$
\left(\mathbf{K}_{0}-\omega^{2} \mathbf{M}_{0}\right) \mathbf{u}=\mathbf{f}
$$

which represents a system of $3 N$ linear equations of motion, where $N$ is the number of FE-model nodes.

\section{Piezoceramic patch}

In the following, the variational formulation for a piezoceramic patch with continuous top and bottom electrodes is derived and a FE-model established, which will later be coupled to the FE-model of the host plate structure.

The mechanical dynamic equilibrium of the piezoceramic patch is described similarly to (1), (2) and (3) as

$$
\begin{array}{rlr}
\sigma_{i j, j}^{p} & =\rho_{p} \ddot{u}_{i}^{p} \quad \text { in } \Omega_{p} \\
\sigma_{i j}^{p} n_{j}^{p} & =F_{i}^{p} & \text { on } \Gamma_{F}^{p} \\
u_{i}^{p} & =\bar{u}_{i}^{p} & \text { on } \Gamma_{u}^{p}
\end{array}
$$

with $\Omega_{p}$ being the patch domain of boundary $\partial \Omega_{p}=\Gamma_{F}^{p} \cup$ $\Gamma_{u}^{p}$ and $\Gamma_{F}^{p} \cap \Gamma_{u}^{p}=\emptyset$, and $\rho_{p}$ the mass density of the piezoceramic patch. Again, it is assumed that no body loads act on the patch domain. Additionally, the electric quasi-static equilibrium and boundary conditions for the piezoceramic patch follow as

$$
\begin{aligned}
D_{i, i} & =0 & & \text { in } \Omega_{p} \\
D_{i} n_{i} & =-Q & & \text { on } \Gamma_{Q} \\
\varphi & =\bar{\varphi} & & \text { on } \Gamma_{\varphi}
\end{aligned}
$$

with the electric displacement $D_{i}$, surface charge density $Q$ on $\Gamma_{Q}$, electric potential $\varphi$ and prescribed potential $\bar{\varphi}$ on $\Gamma_{\varphi}$. Here, the total patch boundary follows as $\partial \Omega_{p}=\Gamma_{Q} \cup \Gamma_{\varphi}$ and $\Gamma_{Q} \cap \Gamma_{\varphi}=\emptyset$.

Again, the weighted residual method is used to obtain the variational formulations, by multiplying the mechanical and electric equilibrium equations (18) and (21) with the virtual displacements $\delta u_{i}^{p}$ and potential $\delta \varphi$, respectively, followed by integration over the patch domain

$$
\begin{aligned}
\int_{\Omega_{p}}\left(\sigma_{i j, j}^{p}-\rho_{p} \ddot{u}_{i}^{p}\right) \delta u_{i}^{p} d \Omega_{p} & =0 \\
\int_{\Omega_{p}} D_{i, i} \delta \varphi d \Omega_{p} & =0
\end{aligned}
$$

The weak variational formulations follow by integration by parts and substitution of the mechanical and electrical kinematically admissible boundary conditions (19) and (22)

$$
\begin{array}{r}
\int_{\Omega_{p}} \delta \varepsilon_{i j}^{p} \sigma_{i j}^{p} d \Omega_{p}+\int_{\Omega_{p}} \delta u_{i}^{p} \rho_{p} \ddot{u}_{i}^{p} d \Omega_{p} \\
=\sum_{i_{c}=1}^{n_{c}} \delta u_{\alpha}^{p}\left(x_{i_{c}}, y_{i_{c}}\right)\left(F_{\alpha}^{p}\right)_{i_{c}} \\
\int_{\Omega_{p}} \delta E_{i} D_{i} d \Omega_{p}=\int_{\Gamma_{Q}} \delta \varphi Q d \Gamma_{Q}
\end{array}
$$

where the fields have to satisfy the corresponding mechanical and electrical essential boundary conditions (20) and (23). It is noted that the boundary loads will be considered only as concentrated loads at $n_{c}$ points of the coordinates $\left(x_{i_{c}}, y_{i_{c}}\right)$, while $\alpha=1,2$ with summation over the repeated indices.

By assuming that the piezoceramic patch is thin and bonded to either of the major plate surfaces, the effect from the patch on the plate approximately reduces to the in-plane electric forces, whereby a plane stress constitutive behaviour, similar to (6), can be used for the patch. This implies that the bending stiffness and associated transverse inertia effects of the patch are not considered. However, when the patch is thin, the bending stiffness of the patch becomes insignificant, while the omission of the transverse inertia effects can be accounted for by lumping the mass of the patch at the relevant dofs in the system mass matrix, which will be discussed in the benchmark examples.

Regarding the electric domain, it is assumed that no electric field is generated from bending of the piezoceramic patch and the electric field is considered to be non-vanishing only in the transverse (poling) direction 3. Hereby, the reduced electromechanical constitutive equations can be written as

$$
\left[\begin{array}{c}
\boldsymbol{\sigma}_{p} \\
D_{3}
\end{array}\right]=\left[\begin{array}{cc}
\frac{E_{p}}{1-\nu_{p}} \mathbf{D}_{p} & -\mathbf{e}_{3} \\
\mathbf{e}_{3}^{T} & \epsilon_{33}
\end{array}\right]\left[\begin{array}{c}
\varepsilon_{p} \\
E_{3}
\end{array}\right]
$$

They represent the relations between the mechanical in-plane stresses $\sigma_{p}$ and transverse electric displacement $D_{3}$, and the associated in-plane strains $\varepsilon_{p}$ and transverse electric field $E_{3}$. The pure mechanical behaviour is recovered by SC patch electrodes, while the pure electric behaviour is represented by the dielectric constant at constant (nil) strains $\epsilon_{33}^{\varepsilon_{p}}$. The electromechanical coupling is governed by the piezoelectric constants in the vector $\mathbf{e}_{3}$, defined for a piezoceramic patch as

$$
\mathbf{e}_{3}=d_{31} \frac{E_{p}}{1-\nu_{p}} \mathbf{D}_{p} \mathbf{b}=e_{31} \mathbf{b}
$$

where $E_{p}$ is the Young's modulus of the piezoceramic material, $\nu_{p}$ is the corresponding Poisson's ratio, and $d_{31} \mathbf{b}=$ 
$d_{31}\left[\begin{array}{lll}1 & 1 & 0\end{array}\right]^{T}$ represents the isotropic plane electromechanical coupling with the strength governed by the piezoelectric strain coefficient $d_{31}=d_{32}$. It is noted that there is no inherent coupling with the in-plane shear strain for piezoceramic materials, which is the reason for the zero component in the last entry of $\mathbf{b}$. Because $1-\nu_{p}$ is contained explicitly in (27), the remaining SC constitutive matrix $\mathbf{D}_{p}$ can be written as in (7) with $\nu_{p}$ instead of $\nu$. It is readily shown that $\mathbf{D}_{p} \mathbf{b}=\mathbf{b}$, whereby the scalar plane stress-reduced piezoelectric coupling coefficient in (27) is identified as

$$
e_{31}=d_{31} \frac{E_{p}}{1-\nu_{p}}
$$

It recovers the stress piezoelectric coupling coefficient resulting from the one-directional (1D) transverse mode constitutive equation when $\nu_{p}=0$.

The in-plane strains can here be represented by

$$
\varepsilon_{p}=\nabla_{p} \boldsymbol{v}_{p}
$$

where the in-plane displacements $u$ and $v$ are arranged in the displacement vector $\boldsymbol{v}_{p}=[u, v]^{T}$, and the 2D derivation operator $\nabla_{p}$ is as in (9). As for the host plate, the frequency domain representation of the weak variational formulations (25) of the patch, is reached by assuming harmonic loads and solutions $\boldsymbol{v}_{p}=\mathbf{v}_{p} \exp (i \omega t)$.

The patch thickness $t_{p}$ is assumed significantly smaller than a characteristic in-plane dimension of the patch surface. Hereby, the electric field $E_{3}$ is approximately constant through the patch thickness and can thus be determined by the difference between the electric potentials on the top $\varphi_{+}$ and bottom $\varphi_{-}$electrodes by

$$
E_{3}=-\nabla_{\varphi} \varphi=-\left[1 / t_{p} \quad-1 / t_{p}\right]\left[\begin{array}{l}
\varphi_{+} \\
\varphi_{-}
\end{array}\right]
$$

Thus, in this notation, the symbol $\nabla_{\varphi}$ denotes the row vector $[1-1] / t_{p}$. By substituting (26), (27), (29) and (30) into (25), the variational weak formulations for the patch (25) can be written in the frequency domain as

$$
\begin{array}{r}
\int_{A_{p}} \delta \mathbf{v}_{p}^{T}\left(\nabla_{p}^{T} \frac{E_{p} t_{p}}{1-\nu_{p}} \mathbf{D}_{p} \boldsymbol{\nabla}_{p} \mathbf{v}_{p}+\nabla_{p}^{T} e_{31} t_{p} \mathbf{b} \nabla_{\varphi} \varphi\right. \\
\left.-\omega^{2} \rho_{p} t_{p} \mathbf{v}_{p}\right) d A_{p}=\sum_{i_{c}=1}^{n_{c}} \delta u_{\alpha}^{p}\left(x_{i_{c}}, y_{i_{c}}\right)\left(F_{\alpha}^{p}\right)_{i_{c}}
\end{array}
$$

for the mechanical relation and as

$$
\begin{aligned}
\int_{A_{p}} \delta \varphi^{T} \nabla_{\varphi}^{T}\left(-e_{31} t_{p} \mathbf{b}^{T} \nabla_{p} \mathbf{v}_{p}\right. & \left.+\epsilon_{33}^{\varepsilon_{p}} t_{p} \boldsymbol{\nabla}_{\varphi} \varphi\right) d A_{p} \\
& =\int_{A_{Q}} \delta \boldsymbol{\varphi}^{T} \boldsymbol{Q} d A_{Q}
\end{aligned}
$$

for the corresponding electrical relation. In both relations, the integration over the constant patch thickness $t_{p}$ has been conducted. From (32), it is seen that the surface charge densities in $\boldsymbol{Q}=\left[Q_{+}, Q_{-}\right]^{T}$ are distributed on the top and bottom electrodes with identical surface areas $A_{Q}$, while zero surface charges are assumed on the lateral parts of the patch boundaries.

The FE-formulation for the piezoceramic patch is then established by introducing a rectangular four node element

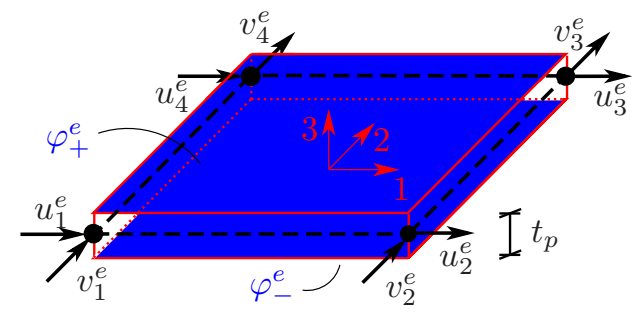

Figure 1. Piezoceramic patch element with 8 mechanical and 2 electric dofs.

with two uniform electric potential surface dofs for the top and bottom electrodes

$$
\varphi=\varphi^{e}=\left[\begin{array}{ll}
\varphi_{+}^{e} & \varphi_{-}^{e}
\end{array}\right]^{T}
$$

and two nodal dofs representing the in-plane displacements $u_{i}$ and $v_{i}$, as shown in Figure 1. The harmonic amplitudes $\mathbf{v}_{p}$ can therefore be represented by

$$
\begin{aligned}
\mathbf{v}_{p}^{e}(x, y) & =\mathbf{N}_{p}(x, y) \mathbf{u}_{p}^{e} \\
& =\left[\begin{array}{ccccc}
N_{1} & 0 & \cdots & N_{4} & 0 \\
0 & N_{1} & \cdots & 0 & N_{4}
\end{array}\right]\left[\begin{array}{c}
u_{1}^{e} \\
v_{1}^{e} \\
\vdots \\
u_{4}^{e} \\
v_{4}^{e}
\end{array}\right]
\end{aligned}
$$

where $\mathbf{N}_{p}$ contains the shape functions $N_{i}, \mathbf{u}_{p}^{e}$ the nodal displacements, while subscript 4 again represents the number of element nodes.

The discretized equations of motion are obtained next by substituting (33) and (34) into both (31) and (32), followed by summation over the number of patch elements $n_{p}$ as

$$
\begin{array}{r}
\sum_{e=1}^{n_{p}}\left[\begin{array}{l}
\delta \mathbf{u}_{p}^{e} \\
\delta \boldsymbol{\varphi}^{e}
\end{array}\right]^{T}\left\{\left[\begin{array}{cc}
\mathbf{K}_{p}^{e}-\omega^{2} \mathbf{M}_{p}^{e} & \mathbf{K}_{c}^{e} \\
-\left(\mathbf{K}_{c}^{e}\right)^{T} & \left(\mathbf{C}_{p}^{\varepsilon_{p}}\right)^{e}
\end{array}\right]\left[\begin{array}{c}
\mathbf{u}_{p}^{e} \\
\boldsymbol{\varphi}^{e}
\end{array}\right]\right. \\
\left.-\left[\begin{array}{c}
\mathbf{f}_{p}^{e} \\
\mathbf{Q}^{e}
\end{array}\right]\right\}=0
\end{array}
$$

In this expression, the element mass, SC stiffness and electromechanical coupling matrices are determined as

$$
\begin{aligned}
\mathbf{M}_{p}^{e} & =\int_{A_{p}^{e}} \mathbf{N}_{p}^{T} \rho_{p} t_{p} \mathbf{I}_{2} \mathbf{N}_{p} d A_{p}^{e} \\
\mathbf{K}_{p}^{e} & =\int_{A_{p}^{e}}\left(\boldsymbol{\nabla}_{p} \mathbf{N}_{p}\right)^{T} \frac{E_{p} t_{p}}{1-\nu_{p}} \mathbf{D}_{p}\left(\boldsymbol{\nabla}_{p} \mathbf{N}_{p}\right) d A_{p}^{e} \\
\mathbf{K}_{c}^{e} & =\int_{A_{p}^{e}}\left(\boldsymbol{\nabla}_{p} \mathbf{N}_{p}\right)^{T} \mathbf{b} e_{31} t_{p} \boldsymbol{\nabla}_{\varphi} d A_{p}^{e}
\end{aligned}
$$

where $\mathbf{I}_{2}$ is the two by two identity matrix, and the element nodal force and surface charge vectors are defined as

$$
\begin{aligned}
\mathbf{f}_{p}^{e} & =\sum_{i_{c}=1}^{n_{c}} \mathbf{N}\left(x_{i_{c}}, y_{i_{c}}\right) \mathbf{f}_{i_{c}}^{p} \\
\mathbf{Q}^{e} & =\int_{A_{p}^{e}}^{\boldsymbol{Q}} \boldsymbol{Q} d A_{p}^{e}
\end{aligned}
$$

with the concentrated force vector $\mathbf{f}_{i_{c}}^{p}=\left[\begin{array}{ll}F_{x}^{p} & F_{y}^{p}\end{array}\right]_{i_{c}}^{T}$. Furthermore, the blocked capacitance matrix in (35) is given 
by

$$
\left(\mathbf{C}_{p}^{\varepsilon_{p}}\right)^{e}=\int_{A_{p}^{e}} \nabla_{\varphi}^{T} \epsilon_{33}^{\varepsilon_{p}} t_{p} \boldsymbol{\nabla}_{\varphi} d A_{p}^{e}=C_{p}^{\varepsilon_{p}}\left[\begin{array}{cc}
1 & -1 \\
-1 & 1
\end{array}\right]
$$

in which

$$
C_{p}^{\varepsilon_{p}}=\frac{\epsilon_{33}^{\varepsilon_{p}} A_{p}}{t_{p}}
$$

is the scalar blocked capacitance.

The global coupled FE-equations for a piezoceramic patch discretized by $n_{p}$ elements are then established by assembly of the element matrices and vectors to the global nodal order, following a rearrangement of the full system matrices and vectors such that the final format of the global FE-equations can be written as

$$
\left[\begin{array}{cc}
\mathbf{K}_{p}-\omega^{2} \mathbf{M}_{p} & \mathbf{K}_{c} \\
-\mathbf{K}_{c}^{T} & \mathbf{C}_{p}^{\varepsilon_{p}}
\end{array}\right]\left[\begin{array}{c}
\mathbf{u}_{p} \\
\boldsymbol{\varphi}_{p}
\end{array}\right]=\left[\begin{array}{c}
\mathbf{f}_{p} \\
\mathbf{Q}
\end{array}\right]
$$

where the mechanical and electric equations are arranged respectively as the first $2 N_{p}$ and last $N_{p e}$ coupled equations of motions; $N_{p}$ is the number of element nodes and $N_{p e}=$ $2 n_{p}$ the number of electrical dofs.

The equipotential condition is then applied to the potentials of the piezoceramic elements forming a continuous surface of an electrode. In the present case, only a single piezoceramic patch is considered with one top and one bottom electrodes, whereby

$$
\varphi_{+}^{1}=\varphi_{+}^{2}=\ldots=\varphi_{+}^{n_{p}} \quad, \quad \varphi_{-}^{1}=\varphi_{-}^{2}=\ldots=\varphi_{-}^{n_{p}}
$$

The equipotential conditions (41) can be imposed by defining a boolean matrix $\mathbf{P}$, that determines the relation between the element potentials in $\varphi_{p}$ and the full surface potentials of the top or bottom electrodes as

$$
\boldsymbol{\varphi}_{p}=\mathbf{P} \boldsymbol{\varphi}_{ \pm}=\left[\begin{array}{lllll}
1 & 0 & \cdots & 1 & 0 \\
0 & 1 & \cdots & 0 & 1
\end{array}\right]^{T}\left[\begin{array}{l}
\varphi_{+} \\
\varphi_{-}
\end{array}\right]
$$

The coupled FE-equations for the discretized piezoceramic patch with consideration of the equipotential condition can thereby be written as

$$
\left[\begin{array}{cc}
\mathbf{K}_{p}-\omega^{2} \mathbf{M}_{p} & \mathbf{K}_{c} \mathbf{P} \\
-\mathbf{P}^{T} \mathbf{K}_{c}^{T} & \mathbf{P}^{T} \mathbf{C}_{p}^{\varepsilon_{p}} \mathbf{P}
\end{array}\right]\left[\begin{array}{c}
\mathbf{u}_{p} \\
\boldsymbol{\varphi}_{ \pm}
\end{array}\right]=\left[\begin{array}{c}
\mathbf{f}_{p} \\
\mathbf{Q}_{ \pm}
\end{array}\right]
$$

which consists of $2 N_{p}+2$ equations of motion, where the charge at the top and bottom electrodes are defined similarly to (42) by

$$
\mathbf{Q}=\mathbf{P Q}_{ \pm} \quad, \quad \mathbf{Q}_{ \pm}=\left[\begin{array}{ll}
\mathbf{Q}_{+} & \mathbf{Q}_{-}
\end{array}\right]^{T}
$$

The introduction of the equipotential conditions results in pure edge coupling between the electric and mechanical domains. The coupling between the equation of motions of the plate (17) and the patch (43) is obtained next by transforming the electric forcing from the patch elements to the equivalent moment loads for the plate elements.

\section{Coupled piezo-plate structure}

Piezoceramic patches are often placed symmetrically in pairs on the host structure. This is beneficial as the neutral axis of the composite and host structure hereby coincide and higher electromechanical coupling can be achieved, when the piezoceramic patches are poled and wired correctly. In the later benchmark examples, only pairs of identical and symmetrically positioned piezoceramic patches are considered. Therefore, a single pair of piezoceramic patches, bonded symmetrically to the upper and lower plate surfaces, is considered in the following. It is assumed that the discretization of the piezoceramic patches concides with the discretization of the underlying part of the plate, whereby the coupling between each (top and bottom) patch and plate element can be described as in Figure 2. It is now considered that the generated electric forcing from the piezoceramic patches acts as external moment loads on the host plate structure, whereby the former force vector $\mathbf{f}$ in (17), can be written as a sum

$$
\mathbf{f}=\mathbf{f}_{e}+\mathbf{f}_{p}^{r}
$$

of the external mechanical loads $\mathbf{f}_{e}$ and the resulting transformed electric forcing from the patches $\mathbf{f}_{p}^{r}$. It is seen from Figure 2 that the in-plane forces $f_{x}^{t}, f_{y}^{t}$ and $f_{x}^{b}, f_{y}^{b}$ of the top and bottom piezoceramic patch elements, respectively, couple with the associated moments $M_{x x}$ and $M_{y y}$ of the plate, through the distance $h$ between the mid planes of the plate and the patches. The moment loads to the plate from the patches in-plane forces can thus be found as

$$
\begin{aligned}
& M_{x x}=-h f_{y}^{t}+h f_{y}^{b} \\
& M_{y y}=h f_{x}^{t}-h f_{x}^{b}
\end{aligned}
$$

Hereby, the resulting electric forcing from the piezoceramic patches $\mathbf{f}_{p}^{r}$ on the plate can be written as

$$
\mathbf{f}_{p}^{r}=\mathbf{W} \mathbf{f}_{p}^{t}-\mathbf{W} \mathbf{f}_{p}^{b}
$$

where $\mathbf{f}_{p}^{t}$ and $\mathbf{f}_{p}^{b}$ are respectively the in-plane forces from (43) of the top and bottom piezoceramic patches transformed through (46) by the connectivity matrix

$$
\mathbf{W}=\left[\begin{array}{ccc}
\mathbf{0} & \mathbf{0} & \mathbf{0} \\
\mathbf{W}_{1} & \mathbf{0} & \mathbf{0} \\
\mathbf{0} & \ddots & \mathbf{0} \\
\mathbf{0} & \mathbf{0} & \mathbf{W}_{N_{p}} \\
\mathbf{0} & \mathbf{0} & \mathbf{0}
\end{array}\right], \mathbf{W}_{i}=\left[\begin{array}{cc}
0 & 0 \\
0 & -h \\
h & 0
\end{array}\right]
$$

for the top patch, while forcing from the bottom patch is determined by $-\mathbf{W}$, as seen in (47). The nodal connectivity array $\mathbf{W}_{i}$ of patch node $i$ is placed at the rows and columns of $\mathbf{W}$ that correspond to the dofs shared by the plate and patches, respectively. Thus, there are as many nodal arrays as there are nodes $\left(N_{p}\right)$ in the piezoceramic patch.

By use of (45), the equation of motion (17) for the plate structure, augmented by the electric forcing from the pair of piezoceramic patches, can then be written as

$$
\left(\mathbf{K}_{0}-\omega^{2} \mathbf{M}_{0}\right) \mathbf{u}-\mathbf{f}_{p}^{r}=\mathbf{f}_{e}
$$

The coupled FE-equations then follow from eliminating the piezoelectric forces $\mathbf{f}_{p}^{r}$ in (49) by (47) and (43), whereby they can be written as

$$
\left[\begin{array}{ccc}
\mathbf{K}-\omega^{2} \mathbf{M} & -\mathbf{W K}_{c} \mathbf{P} & \mathbf{W} \mathbf{K}_{c} \mathbf{P} \\
\left(\mathbf{W K} \mathbf{K}_{c} \mathbf{P}\right)^{T} & \mathbf{P}^{T} \mathbf{C}_{p}^{\varepsilon_{p}} \mathbf{P} & \mathbf{0} \\
-\left(\mathbf{W K} \mathbf{K}_{c} \mathbf{P}\right)^{T} & \mathbf{0} & \mathbf{P}^{T} \mathbf{C}_{p}^{\varepsilon_{p}} \mathbf{P}
\end{array}\right]\left[\begin{array}{c}
\mathbf{u} \\
\boldsymbol{\varphi}_{ \pm}^{t} \\
\boldsymbol{\varphi}_{ \pm}^{b}
\end{array}\right]=\left[\begin{array}{c}
\mathbf{f}_{e} \\
\mathbf{Q}_{ \pm}^{t} \\
\mathbf{Q}_{ \pm}^{b}
\end{array}\right]
$$




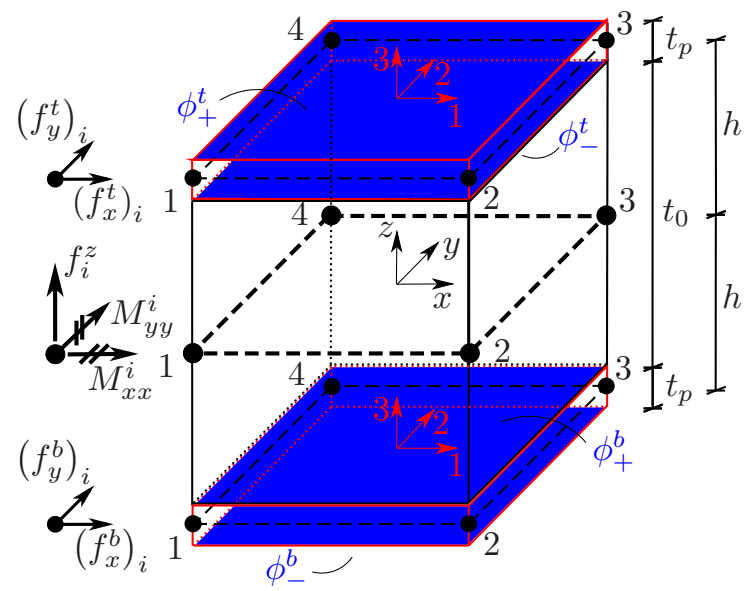

Figure 2. Moment equilibrium of plate and piezoceramic patch elements, superscript $e$ is omitted in the figure notations.

which consists of the full set of $3 N$ mechanical equations and four electric equations for the pair of piezoceramic patches. The mass and stiffness matrices of the piezoceramic patches further provide additional terms in the system matrices,

$$
\mathbf{K}=\mathbf{K}_{0}+2 \mathbf{W} \mathbf{K}_{p} \mathbf{W}^{T}, \quad \mathbf{M}=\mathbf{M}_{0}+2 \mathbf{W} \mathbf{M}_{p} \mathbf{W}^{T}
$$

where the multiplication with the connectivity matrix $\mathbf{W}$ ensures that the mass and stiffness effects of the patches are transferred to the correct nodes of the plate structure.

It is now the aim to reduce the number of electric equations by considering the pair of patches being either configured as SP-PW or OP-SW and connected to a shunt circuit with an impedance $Z_{s h}(\omega)$, see Figure 3.

SP-PW pair of patches For the SP-PW shunted pair of piezoceramic patches, the following relations between the electrodes potentials can be deduced from Figure 3(a) as

$$
\varphi_{-}^{t}=\varphi_{+}^{b} \quad, \quad \varphi_{+}^{t}=\varphi_{-}^{b} \quad, \quad V=\varphi_{+}^{t}-\varphi_{-}^{t}
$$

These relations are now used to eliminate some potentials and to introduce the voltage $V$ over the electric shunt by establishing the matrix $\mathbf{P}_{\varphi}$ as follows

$$
\left[\begin{array}{c}
\boldsymbol{\varphi}_{ \pm}^{t} \\
\boldsymbol{\varphi}_{ \pm}^{b}
\end{array}\right]=\mathbf{P}_{\varphi}\left[\begin{array}{c}
V \\
\varphi_{-}^{t} \\
\boldsymbol{\varphi}_{ \pm}^{b}
\end{array}\right], \quad \mathbf{P}_{\varphi}=\left[\begin{array}{cccc}
1 & 1 & 0 & 0 \\
0 & 1 & 0 & 0 \\
0 & 1 & 0 & 0 \\
1 & 1 & 0 & 0
\end{array}\right]
$$

Introducing (53) in (50), the coupling and capacitance matrices are altered by $\mathbf{P}_{\varphi}$, such that

$$
\begin{aligned}
& \mathbf{P}_{\varphi}^{T}\left[\begin{array}{cc}
\mathbf{P}^{T} \mathbf{C}_{p}^{\varepsilon_{p}} \mathbf{P} & \mathbf{0} \\
\mathbf{0} & \mathbf{P}^{T} \mathbf{C}_{p}^{\varepsilon_{p}} \mathbf{P}
\end{array}\right] \mathbf{P}_{\varphi}=2 C_{p}^{\varepsilon_{p}}\left[\begin{array}{l}
1 \\
\mathbf{0}
\end{array}\right]\left[\begin{array}{ll}
1 & \mathbf{0}
\end{array}\right] \\
& \mathbf{W}\left[\begin{array}{ll}
-\mathbf{K}_{c} \mathbf{P} & \mathbf{K}_{c} \mathbf{P}
\end{array}\right] \mathbf{P}_{\varphi}=-\mathbf{W k}_{c} 2 e_{31}\left[\begin{array}{ll}
1 & \mathbf{0}
\end{array}\right]
\end{aligned}
$$

Thus, the capacitance is determined by a scalar $2 C_{p}^{\varepsilon_{p}}$ for the parallel wired pair of patches and the coupling by a vector $\mathbf{W} \mathbf{k}_{c} 2 e_{31}$ as the rows and arrays associated with the remaining potentials contain zeroes only. It is noted that the blocked capacitance $C_{p}^{\varepsilon_{p}}$ is given in (39) and the coupling (a)
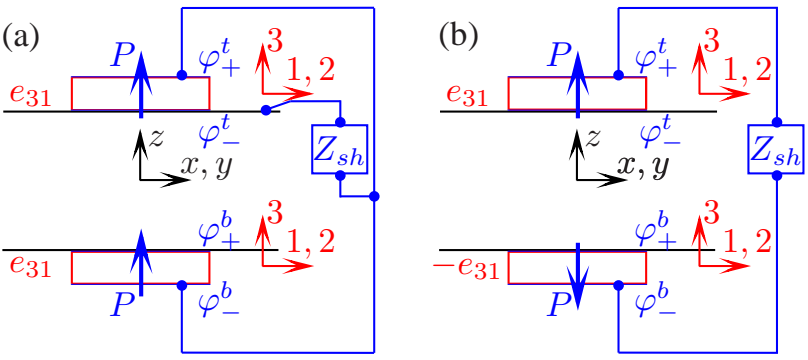

Figure 3. Conducting plate structure with a pair of patches wired to a shunt circuit according to poling configurations (a) SP-PW and (b) OP-SW.

vector $\mathbf{k}_{c}$ is defined as

$$
\mathbf{k}_{c}=\mathbf{K}_{c} \mathbf{P}\left[\begin{array}{c}
1 \\
-1
\end{array}\right] \frac{1}{2 e_{31}}
$$

By defining a resulting capacitance $\bar{C}_{p}^{\varepsilon_{p}}$ and plane stressreduced piezoelectric coupling coefficient $\bar{e}_{31}$ as

$$
\bar{C}_{p}^{\varepsilon_{p}}=2 C_{p}^{\varepsilon_{p}} \quad, \quad \bar{e}_{31}=2 e_{31},
$$

the coupled FE-equations (50) can be reduced to

$$
\left[\begin{array}{cc}
\mathbf{K}-\omega^{2} \mathbf{M} & -\mathbf{W k}_{c} \bar{e}_{31} \\
\left(\mathbf{W k}_{c} \bar{e}_{31}\right)^{T} & \bar{C}_{p}^{\varepsilon_{p}}
\end{array}\right]\left[\begin{array}{c}
\mathbf{u} \\
V
\end{array}\right]=\left[\begin{array}{c}
\mathbf{f}_{e} \\
\mathbf{Q}
\end{array}\right]
$$

where the number of electric equations is reduced to one with the voltage as variable, while the resulting charge

$$
\mathrm{Q}=\mathrm{Q}_{+}^{t}+\mathrm{Q}_{-}^{b}
$$

is obtained after multiplication of the surface charges in (50) with $\mathbf{P}_{\varphi}^{T}$.

The resulting charge $\mathrm{Q}$ can be linked to the voltage $V$ through the shunt impedance $Z(\omega)$ via Ohm's law

$$
V=-i \omega Z_{s h}(\omega) \mathrm{Q}
$$

Therefore, the coupled FE-equations for the plate with a pair of SP-PW shunted piezoceramic patches can be written as

$$
\left[\begin{array}{cc}
\mathbf{K}-\omega^{2} \mathbf{M} & -\mathbf{W} \mathbf{k}_{c} \bar{e}_{31} \\
\left(\mathbf{W k}_{c} \bar{e}_{31}\right)^{T} & \bar{C}_{p}^{\varepsilon_{p}}+\frac{1}{i \omega Z_{s h}(\omega)}
\end{array}\right]\left[\begin{array}{c}
\mathbf{u} \\
V
\end{array}\right]=\left[\begin{array}{c}
\mathbf{f}_{e} \\
0
\end{array}\right]
$$

where the inverse of the shunt impedance enters in the last diagonal term of the system matrix after substitution of (59) into (57) leading to the zero on the right hand side of (60).

OP-SW pair of patches For the OP-SW shunted pair of patches in Figure 3(b), the relations between the potentials can be defined as

$$
\varphi_{-}^{t}=\varphi_{+}^{b} \quad, \quad V=\varphi_{+}^{t}-\varphi_{-}^{b}
$$

and furthermore, as the patches are considered identical and symmetrically positioned on the host structure, the individual differences in patches potentials are equal

$$
\varphi_{+}^{t}-\varphi_{-}^{t}=\varphi_{+}^{b}-\varphi_{-}^{b}
$$


These relations are now defined by the matrix $\mathbf{P}_{\varphi}$ as

$$
\left[\begin{array}{c}
\boldsymbol{\varphi}_{ \pm}^{t} \\
\boldsymbol{\varphi}_{ \pm}^{b}
\end{array}\right]=\mathbf{P}_{\varphi}\left[\begin{array}{c}
V \\
\varphi_{-}^{t} \\
\boldsymbol{\varphi}_{ \pm}^{b}
\end{array}\right], \quad \mathbf{P}_{\varphi}=\left[\begin{array}{cccc}
1 / 2 & 1 & 0 & 0 \\
0 & 1 & 0 & 0 \\
0 & 1 & 0 & 0 \\
-1 / 2 & 1 & 0 & 0
\end{array}\right]
$$

whereby the capacitance and coupling matrices are altered by the substitution of (63) into (50),

$$
\begin{array}{r}
\mathbf{P}_{\varphi}^{T}\left[\begin{array}{cc}
\mathbf{P}^{T} \mathbf{C}_{p}^{\varepsilon_{p}} \mathbf{P} & \mathbf{0} \\
\mathbf{0} & \mathbf{P}^{T} \mathbf{C}_{p}^{\varepsilon_{p}} \mathbf{P}
\end{array}\right] \mathbf{P}_{\varphi}=\frac{1}{2} C_{p}^{\varepsilon_{p}}\left[\begin{array}{l}
1 \\
\mathbf{0}
\end{array}\right]\left[\begin{array}{ll}
1 & \mathbf{0}
\end{array}\right] \\
\mathbf{W}\left[\begin{array}{ll}
-\mathbf{K}_{c} \mathbf{P} & -\mathbf{K}_{c} \mathbf{P}
\end{array}\right] \mathbf{P}_{\varphi}=-\mathbf{W} \mathbf{k}_{c} e_{31}\left[\begin{array}{ll}
1 & \mathbf{0}
\end{array}\right]
\end{array}
$$

As for the SP-PW pair of patches, the number of electric equations can be reduced to one with the voltage as variable, whereby (50) can be written as (60), now with the resulting capacitance and plane stress-reduced piezoelectric coupling coefficient defined for the OP-SW pair of patches as

$$
\bar{C}_{p}^{\varepsilon_{p}}=\frac{1}{2} C_{p}^{\varepsilon_{p}} \quad, \quad \bar{e}_{31}=e_{31}
$$

It is seen that the resulting capacitances for the SP-PW and OP-SW pairs of piezoceramic patches correspond to the resulting capacitances of two capacitors connected, respectively, in parallel and series, see Chevallier et al. (2009). For both configurations of the pairs of piezoceramic patches the vector

$$
\mathbf{w}=\mathbf{W k}_{c}
$$

defines a resulting scalar measure of displacement $u_{p}$ of the pair of patches as

$$
u_{p}=\mathbf{w}^{T} \mathbf{u}
$$

used in the following.

\section{Shunt circuit}

In the coupled FE-equations (60), the voltage $V$ is governed by the relation between the resulting blocked capacitance $\bar{C}_{p}^{\varepsilon_{p}}$ and plane stress-reduced piezoelectric coupling coefficient $\bar{e}_{31}$ and the shunt circuit impedance $Z_{s h}(\omega)$. The former can be obtained from the material properties, electrode configuration and wiring of the pair of piezoceramic patches, while the shunt circuit impedance depends on the configuration and tuning of the connected shunt electronic components.

The solution to (60) is bounded by two limits associated with SC and OC patch electrodes. The corresponding SC and $\mathrm{OC}$ eigenvalue problems can be deduced from (57), with respectively $V=0$ and $\mathrm{Q}=0$. The $\mathrm{SC}$ eigenvalue problem hereby follows as

$$
\left[\mathbf{K}-\omega_{j}^{2} \mathbf{M}\right] \mathbf{u}_{j}=\mathbf{0}
$$

with $\mathrm{SC}$ frequencies $\omega_{j}$ and mode shapes $\mathbf{u}_{j}$, while the additional sensor equation provides the modal charge

$$
\mathrm{Q}_{j}=-\bar{e}_{31} \mathbf{w}^{T} \mathbf{u}_{j}
$$

In the opposite OC limit, the stiffness is augmented by the voltage stiffening contribution which, for $\mathrm{Q}=0$, can be determined as

$$
V=\frac{\bar{e}_{31}}{\bar{C}_{p}^{\varepsilon_{p}}} \mathbf{w}^{T} \mathbf{u}
$$

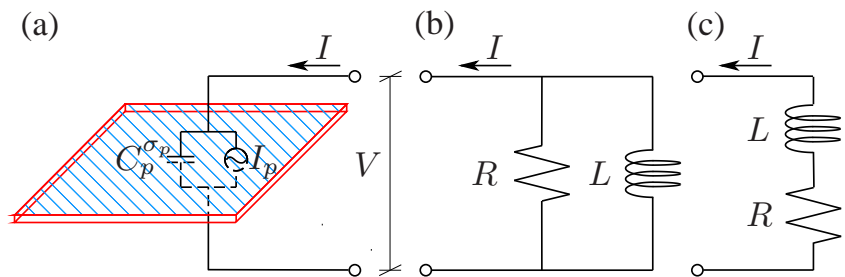

Figure 4. Electric model for (a) piezoceramic patch, with (b) parallel and (c) series $R L$ shunts, where $I$ is the electric current and $C_{p}^{\sigma_{p}}$ is the constant stress (free) capacitance.

Inserting this relation in the former mechanical equations of (57), the OC eigenvalue problem is determined as

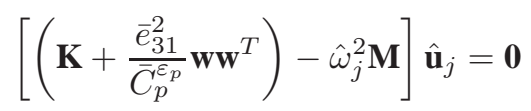

Thus, the OC circular frequencies $\hat{\omega}_{j} \geq \omega_{j}$ because of the piezoelectric effect, where the equality might occur due to charge cancelation effects.

The relative difference between the squared OC and SC frequencies provides the so-called squared effective EMCC

$$
\kappa_{e}^{2}=\frac{\hat{\omega}_{j}^{2}-\omega_{j}^{2}}{\omega_{j}^{2}}
$$

which determines the authority of the pair of patches on mode $j$ and consequently the associated attainable modal damping from the supplemental shunt. Therefore, the latter increases with the separation of the two limiting natural frequencies $\omega_{j}$ and $\hat{\omega}_{j}$.

For $R L$ shunts, the inductance $L$ is calibrated in order for the circuit frequency to work in resonance with the vibrating structure, while the resistance $R$ is tuned in order to maximize the dissipation of electrical energy. The $R L$ network can be established by either placing the inductance and resistance in parallel or in series, as shown in Figure 4. It is noted that these networks are idealized in the sense that any resistance associated with either synthetic or purely passive inductors is neglected, which may have an effect on the tuning of the shunt resistance. Further, it can be seen in Figure 4 that the capacitance is the stress free capacitance $C_{p}^{\sigma_{p}}$, which can be found by the relation to the blocked capacitance for the transverse response mode as

$$
C_{p}^{\varepsilon_{p}}=C_{p}^{\sigma_{p}}\left(1-k_{31}^{2}\right) \quad, \quad k_{31}^{2}=2 \frac{d_{31} e_{31}}{\epsilon_{33}^{\sigma_{p}}}
$$

where $k_{31}$ is the piezoceramic material plane stress-reduced electromechanical coupling factor.

For the parallel shunt configuration in Figure 4(b), the impedance $Z_{s h}(\omega)$ is conveniently expressed in terms of reciprocal values,

$$
\frac{1}{Z_{s h}(\omega)}=\frac{1}{R}+\frac{1}{i \omega L}
$$

Substitution of the previous impedance function into (60) gives the parallel coupled FE-equations

$$
\left[\begin{array}{cc}
\mathbf{K}-\omega^{2} \mathbf{M} & -\mathbf{w} \bar{e}_{31} \\
\bar{e}_{31} \mathbf{w}^{T} & \bar{C}_{p}^{\varepsilon_{p}}+\frac{1}{i \omega R}-\frac{1}{\omega^{2} L}
\end{array}\right]\left[\begin{array}{c}
\mathbf{u} \\
V
\end{array}\right]=\left[\begin{array}{c}
\mathbf{f}_{e} \\
0
\end{array}\right]
$$


with the inverse impedance terms appearing directly in the system matrix.

For the series network in Figure 4(c), the impedance function is given as

$$
Z_{s h}(\omega)=R+i \omega L
$$

whereby the series coupled FE matrix equation takes the form

$$
\left[\begin{array}{cc}
\mathbf{K}-\omega^{2} \mathbf{M} & -\mathbf{w} \bar{e}_{31} \\
\bar{e}_{31} \mathbf{w}^{T} & \bar{C}_{p}^{\varepsilon_{p}}+\frac{1}{i \omega R-\omega^{2} L}
\end{array}\right]\left[\begin{array}{c}
\mathbf{u} \\
V
\end{array}\right]=\left[\begin{array}{c}
\mathbf{f}_{e} \\
0
\end{array}\right]
$$

For both shunt circuit configurations, the mechanical equivalence is presented in e.g. Høgsberg and Krenk (2017), where the inerter (inductance) and the damper (resistance) are connected in series for the parallel $R L$ shunt, while they are placed in parallel for the series shunt. However, in the present work the electric notation is kept, whereby existing shunt calibration formulas can be directly applied based on the derived electric analogies.

\section{Shunt Tuning Based on Balanced Modal Calibration}

In this second section, the coupled FE-equations (75) and (77) for the parallel and series shunt circuits are analyzed in order to determine the particular shunt tuning that maximizes the damping of a targeted resonant vibration mode $j=r$. In order to apply analytical calibration methods, the full complex eigenvalue problem is reduced to a representative system of only two coupled equations, governing the structural response of target mode $r$ and the associated electric loading from the pair of piezoceramic patches.

The mechanical equations in (75) and (77) can be decoupled in terms of the mode shapes $\mathbf{u}_{j}$ from the SC eigenvalue problem in (68). For this purpose, the displacement vector $\mathbf{u}$ is represented by a linear combination of the normalized mode shape vectors $\mathbf{u}_{j} /\left(\mathbf{w}^{T} \mathbf{u}_{j}\right)$, whereafter pre-multiplication of (75) and (77), with the transpose of the normalized mode shape vector gives the scalar equation of motion

$$
\left(k_{j}-\omega^{2} m_{j}\right) u_{j}-\bar{e}_{31} V=f_{j}
$$

in which the normalised modal mass, stiffness and loads are defined as

$$
m_{j}=\frac{\mathbf{u}_{j}^{T} \mathbf{M} \mathbf{u}_{j}}{\left(\mathbf{w}^{T} \mathbf{u}_{j}\right)\left(\mathbf{u}_{j}^{T} \mathbf{w}\right)}, \quad k_{j}=\frac{\mathbf{u}_{j}^{T} \mathbf{K} \mathbf{u}_{j}}{\left(\mathbf{w}^{T} \mathbf{u}_{j}\right)\left(\mathbf{u}_{j}^{T} \mathbf{w}\right)}, \quad f_{j}=\frac{\mathbf{u}_{j}^{T} \mathbf{f}_{e}}{\mathbf{w}^{T} \mathbf{u}_{j}}
$$

The resulting displacement $u_{p}$ (67) of the pair of patches is, because of the normalisation, given by the sum of the modal displacements $u_{j}$ obtained by solving the $3 N$ scalar equations (78).

The aim is to derive the optimal shunt tuning for a specific resonant vibration mode $j=r$, which can be approximated by the single dynamic term $j=r$ and two supplemental terms, accounting for the flexibility and inertia effects from the non-resonant modes $j \neq r$ (Krenk and Høgsberg, 2016). This modal correction approach is based on a two-term representation of the response contribution from non-resonant modes and enables the derivation of explicit expressions for the optimal shunt tuning. This is briefly reviewed in the following and expressed in terms of representative electro-mechanical components.

The resulting plane stress-reduced piezoelectric coupling coefficient $\bar{e}_{31}$ translates displacement in the mechanical domain to charge Q in the electric domain by (69). This is now used to eliminate $u_{j}$ in the modal equations of motion (78), which by considering free vibrations can be written as

$$
-\left(\frac{k_{j}}{\bar{e}_{31}^{2}}-\omega^{2} \frac{m_{j}}{\bar{e}_{31}^{2}}\right) \mathrm{Q}_{j}=V
$$

The sum of the modal charge components $\mathrm{Q}_{j}$ determines the magnitude of the resulting charge $\mathrm{Q}$.

For resonant damping, the charge $Q$ is specifically determined at the resonant frequency of mode $r$, taking into account the influence from the other residual modes by including the flexibility and inertia correction terms as presented in Krenk and Høgsberg (2016) and transferred to the equivalent electric corrections by multiplication with $\bar{e}_{31}^{2}$,

$$
\begin{array}{rcc}
C_{r}^{\prime} & = & \bar{e}_{31}^{2} \mathbf{w}^{T} \mathbf{K}_{r}^{-1} \mathbf{K K}_{r}^{-1} \mathbf{w}-\frac{\bar{e}_{31}^{2}}{k_{r}} \\
\frac{\omega_{r}^{2}}{L_{r}^{\prime}}= & \bar{e}_{31}^{2} \mathbf{w}^{T} \mathbf{K}_{r}^{-1} \mathbf{K} \mathbf{K}_{r}^{-1} \mathbf{w}-\bar{e}_{31}^{2} \mathbf{w}^{T} \mathbf{K}_{r}^{-1} \mathbf{w}
\end{array}
$$

The modified stiffness and mass matrices correspond to removing the mass contribution from the resonant mode and making a frequency shift of the stiffness matrix. They are given as, Krenk and Høgsberg (2016),

$$
\mathbf{K}_{r}=\mathbf{K}-\omega_{r}^{2} \mathbf{M}_{r} \quad, \quad \mathbf{M}_{r}=\mathbf{M}-\frac{\left(\mathbf{M} \mathbf{u}_{r}\right)\left(\mathbf{M} \mathbf{u}_{r}\right)^{T}}{\mathbf{u}_{r}^{T} \mathbf{M} \mathbf{u}_{r}}
$$

with $\mathbf{K}_{r}=\mathbf{K}$ for the case of a pure quasi-static residual mode correction. The flexibility and inertia corrections for the nonresonant modes, due to the presence of the electric shunt, can thus be represented by the modal capacitance $C_{r}^{\prime}$ and inductance $L_{r}^{\prime}$ in (81), respectively. Hereby, the charge Q is truncated consistently for mode $r$ as

$$
\mathrm{Q} \simeq \mathrm{Q}_{r}-\left(C_{r}^{\prime}-\frac{1}{\omega^{2} L_{r}^{\prime}}\right) V
$$

where the modal charge for resonant mode $r$ is determined from (80) by the dynamic equation

$$
\mathrm{Q}_{r}=-\frac{\omega_{r}^{2}}{\omega_{r}^{2}-\omega^{2}} \frac{\bar{e}_{31}^{2}}{k_{r}} V
$$

Substitution of the modal electric representation (83) into the last equation of (60) gives the homogeneous equation

$$
\left(\frac{\omega_{r}^{2}}{\omega_{r}^{2}-\omega^{2}} \frac{\bar{e}_{31}^{2}}{k_{r}}+C_{r}-\frac{1}{\omega^{2} L_{r}^{\prime}}+\frac{1}{i \omega Z_{s h}(\omega)}\right) V=0
$$

when the modal charge $Q_{r}$ has been eliminated by (84), while the modal capacitance

$$
C_{r}=\bar{C}_{p}^{\varepsilon_{p}}+C_{r}^{\prime}
$$

is conveniently represented as the sum of the resulting blocked capacitance $\bar{C}_{p}^{\varepsilon_{p}}$ and modal capacitance $C_{r}^{\prime}$ in (81a). 
Non-trivial solutions require the expression inside the parenthesis in (85) to vanish, constituting the characteristic equation of the system. In common calibration methods, based on single mode representations of the mechanical structure, division with the blocked capacitance $\bar{C}_{p}^{\varepsilon_{p}}$ introduces the so-called squared modal EMCC

$$
\kappa_{0}^{2}=\frac{\bar{e}_{31}^{2}}{\bar{C}_{p}^{\varepsilon_{p}} k_{r}}
$$

see for instance Thomas et al. (2012). In the present case, the introduced residual mode corrections in (81) modify the effective capacitance of the pair of piezoceramic patches by a constant flexibility and a frequency dependent inertia contribution from the non-resonant modes. This means that the effective capacitance varies with the frequency as discussed in Berardengo et al. (2016). At resonance in mode $r$, the effective capacitance can thus be determined as

$$
C_{L}=C_{r}-\frac{1}{\omega_{r}^{2} L_{r}^{\prime}}
$$

whereby the residual mode corrected squared modal EMCC can be defined as

$$
\kappa_{L}^{2}=\frac{\bar{e}_{31}^{2}}{C_{L} k_{r}}
$$

As demonstrated in the later benchmark examples, this residual mode corrected modal EMCC approximately equals the effective EMCC in (72), with a minor deviation due to the approximation of the non-resonant mode representation in Krenk and Høgsberg (2016). However, for the derivation of the shunt tuning formulas, it is convenient to introduce the frequency independent squared modal EMCC

$$
\kappa_{r}^{2}=\frac{\bar{e}_{31}^{2}}{C_{r} k_{r}}
$$

which is only modified by the flexibility correction for the non-resonant modes, by the modified capacitance in (86). Hereby, the characteristic equation for (85) can be written as

$$
\frac{\omega_{r}^{2}}{\omega_{r}^{2}-\omega^{2}} \kappa_{r}^{2}+\frac{1}{i \omega Z_{r}(\omega) C_{r}}+1=0
$$

in which the modal shunt circuit impedance

$$
\frac{1}{Z_{r}(\omega)}=\frac{1}{Z_{s h}(\omega)}+\frac{1}{i \omega L_{r}^{\prime}}
$$

is explicitly modified by the modal inductance $L_{r}^{\prime}$ in (81).

\section{Parallel shunt}

For the parallel shunt circuit, the impedance function $Z_{s h}(\omega)$ in (74) is substituted into (92), whereby the modal impedance function can be written as

$$
\frac{1}{Z_{r}(\omega)}=\frac{1}{R_{r}}+\frac{1}{i \omega L_{r}}
$$

introducing the modal inductance and resistance as

$$
\frac{1}{L_{r}}=\frac{1}{L}+\frac{1}{L_{r}^{\prime}} \quad, \quad R_{r}=R
$$

Any preferred calibration procedure can now be used to determine the modal inductance $L_{r}$ and resistance $R_{r}$. In the
Table 1. Balanced calibration procedure for parallel and series shunt circuits, without $L_{0}, R_{0}$, with flexibility $L_{r}, R_{r}$ and with flexibility-inertia $L, R$ residual mode corrections.

\begin{tabular}{ccc} 
Parameter & Parallel & Series \\
\hline$L_{0}$ & $\frac{1}{\bar{C}_{p}^{\varepsilon_{p}} \omega_{r}^{2}}$ & $\frac{1}{\bar{C}_{p}^{\varepsilon_{p}}\left(1+\kappa_{0}^{2}\right)^{2} \omega_{r}^{2}}$ \\
$R_{0}$ & $\frac{1}{\bar{C}_{p}^{\varepsilon_{p}} \omega_{r}} \sqrt{\frac{1}{2 \kappa_{0}^{2}}}$ & $\frac{1}{\bar{C}_{p}^{\varepsilon_{p}} \omega_{r}} \sqrt{\frac{2 \kappa_{0}^{2}}{\left(1+\kappa_{0}^{2}\right)^{3}}}$ \\
\hline$L_{r}$ & $\frac{1}{C_{r} \omega_{r}^{2}}$ & $\frac{1}{C_{r}\left(1+\kappa_{r}^{2}\right)^{2} \omega_{r}^{2}}$ \\
$R_{r}$ & $\frac{1}{C_{r} \omega_{r}} \sqrt{\frac{1}{2 \kappa_{r}^{2}}}$ & $\frac{1}{C_{r} \omega_{r}} \sqrt{\frac{2 \kappa_{r}^{2}}{\left(1+\kappa_{r}^{2}\right)^{3}}}$ \\
\hline$L$ & $\frac{1}{C_{L} \omega_{r}^{2}}$ & $\frac{1}{C_{L}\left(1+\kappa_{L}^{2}\right)^{2} \omega_{r}^{2}}$ \\
$R$ & $R_{r}$ & $R_{r}\left(L / L_{r}\right)^{2}$ \\
\hline
\end{tabular}

present case, the modal shunt components are determined by the balanced calibration method (Høgsberg and Krenk, 2017), based on the equal modal damping calibration for the tuned mass damper (Krenk , 2005). The electric components of the modal shunt circuit are therefore determined according to Høgsberg and Krenk (2012) by the two calibration formulas

$$
L_{r} C_{r} \omega_{r}^{2}=1 \quad, \quad R_{r} C_{r} \omega_{r}=\sqrt{\frac{1}{2 \kappa_{r}^{2}}}
$$

where $\omega_{r}$ is the natural frequency of the targeted mode with SC electrodes, $C_{r}$ is the modal capacitance in (86), while $\kappa_{r}$ has been defined in (90).

The actual shunt inductance $L$ follows from (94) and is conveniently written as in (95)

$$
L C_{L} \omega_{r}^{2}=1
$$

with the effective modal capacitance defined in (88). Hereby, the resistance in (95) and the inductance in (96) are determined by expressions similar to those for an idealized single-mode structure (Høgsberg and Krenk, 2012), but with modified capacitances $C_{r}$ and $C_{L}$, respectively.

The calibration formulas for $L_{r}, R_{r}$ with only flexibility correction and for $L, R$ with the full flexibility-inertia residual mode correction are listed in the second column of Table 1. Furthermore, the table provides the commonly used single-mode calibration $\left(L_{0}, R_{0}\right)$ determined by the squared modal EMCC in (87) and resulting blocked capacitance $\bar{C}_{p}^{\varepsilon_{p}}$ of the pair of piezoceramic patches.

\section{Series shunt}

For the series shunt circuit, the modal impedance $Z_{r}(\omega)$ is obtained by substitution of (76) into (92)

$$
Z_{r}(\omega)=\frac{i \omega L_{r}^{\prime}(R+i \omega L)}{i \omega L_{r}^{\prime}+(R+i \omega L)}
$$


As for the parallel shunt, this modal function is approximated by the format

$$
Z_{r}(\omega)=R_{r}+i \omega L_{r}
$$

similar to the actual shunt in (76). The modal impedance in (97) is separated into its real and imaginary parts, whereby the frequency-independent $R_{r}$ and $L_{r}$ are obtained by omitting terms containing $R^{2}$. The electrical components of the modal shunt impedance are then obtained as

$$
\frac{1}{L_{r}}=\frac{1}{L}+\frac{1}{L_{r}^{\prime}} \quad, \quad R_{r}=\frac{R}{\left(1+L / L_{r}^{\prime}\right)^{2}}
$$

where the inductance $L_{r}$ is defined as for the parallel shunt in (94), while the modal resistance $R_{r}$ is additionally affected by the modal inductance correction $L_{r}^{\prime}$. Again, the balanced calibration in Høgsberg and Krenk (2012) is applied, which in the present notation gives the following calibration formulas for the modal components of the series shunt,

$$
L_{r} C_{r} \omega_{r}^{2}=\frac{1}{\left(1+\kappa_{r}^{2}\right)^{2}} \quad, \quad R_{r} C_{r} \omega_{r}=\sqrt{\frac{2 \kappa_{r}^{2}}{\left(1+\kappa_{r}^{2}\right)^{3}}}
$$

The actual shunt tuning then follows by considering (88), (89), (99) and (100)

$$
L C_{L} \omega_{r}^{2}=\frac{1}{\left(1+\kappa_{L}^{2}\right)^{2}+\kappa_{L}^{2} \xi} \quad, \quad R=R_{r}\left(\frac{L}{L_{r}}\right)^{2}
$$

which is seen to depend on the residual mode corrected squared modal EMCC, while the parameter $\xi$, in the inductance tuning formula, represents the difference between $\kappa_{r}^{2}$ and $\kappa_{L}^{2}$,

$$
\xi=\kappa_{r}^{2}-\kappa_{L}^{2}=\kappa_{r}^{2} \frac{1}{1-\omega_{r}^{2} L_{r}^{\prime} C_{r}}
$$

The parameter $\xi$ depends on the difference between unity and the ratio of the modal capacitance $C_{r}$ to the inertia correction $1 /\left(\omega_{r}^{2} L_{r}^{\prime}\right)$, and therefore $\xi \rightarrow 0$ when the inertia correction becomes small relative to the modal capacitance. As $\xi$ in (101) is furthermore multiplied by $\kappa_{L}^{2}$, the term is negligible and can be omitted in the tuning of the series connected inductance. The optimal calibration formulas both with and without the flexibility and flexibility-inertia residual mode corrections are summarized in last column of Table 1.

\section{Shunt Tuning Based on the Effective EMCC}

The squared effective EMCC $\kappa_{e}^{2}$ defined in (72) is commonly used as the governing parameter in the calibration of resonant shunt circuits. It is a convenient parameter, as it can be determined experimentally by simple dynamic tests in the SC and $\mathrm{OC}$ limits and thereby takes actual device imperfections into account. However, the squared effective EMCC is typically represented by the squared modal EMCC $\kappa_{0}^{2}$ in (87), evaluated for a single-mode of the structure without any residual mode correction. As demonstrated in the later analysis of two benchmark examples, the approximation $\kappa_{0}^{2} \simeq \kappa_{e}^{2}$ becomes rather inaccurate when the contribution from non-resonant modes is substantial. In particular, the flexibility contribution from the residual modes can be shown to have a significant effect on the evaluated squared modal EMCC $\kappa_{r}^{2}$ in (90), as also discussed by Berardengo et al. (2016). An even more accurate evaluation of the squared effective EMCC is however obtained by the squared modal EMCC $\kappa_{L}^{2}$ in (89), where the modified capacitance $C_{L}$ in (88) contains both the flexibility correction by $C_{r}$ in (86) and the inertia correction directly by $L_{r}^{\prime}$. The only approximation associated with this coupling coefficient is the truncation introduced in the derivation of the residual mode components in (81), see details in Krenk and Høgsberg (2016). Thus, it is investigated in the next section by two benchmark examples how accurately the representation of the squared effective EMCC by

$$
\kappa_{L}^{2} \simeq \kappa_{e}^{2}
$$

captures the influence from residual vibration modes. Assuming $\kappa_{L}^{2}=\kappa_{e}^{2}$, an alternative calibration procedure based on the SC and OC frequencies of the structure can then be established, as demonstrated in the following.

The characteristic equation resulting from (85) is now expressed in terms of the squared effective EMCC $\kappa_{e}^{2}$ and the modified capacitance $C_{L}$ via elimination of $\bar{e}_{31}^{2} / k_{r}$ by (89) and (103), and of $L_{r}^{\prime}$ by (88). Hereby, the characteristic equation can be written as

$\frac{\kappa_{e}^{2} \omega_{r}^{2} \omega^{2}}{\omega_{r}^{2}-\omega^{2}}+\omega_{r}^{2}-\frac{C_{r}}{C_{L}}\left(\omega_{r}^{2}-\omega^{2}\right)+\frac{\omega^{2}}{i \omega Z_{s h}(\omega) C_{L}}=0$

The optimal shunt inductance is now based on the squared effective EMCC $\kappa_{e}^{2}$, while the resistance is subsequently derived from the squared modal EMCC $\kappa_{r}^{2}$. Thus, the present calibration procedure depends on two effective coupling coefficients: $\kappa_{e}^{2}$ and $\kappa_{r}^{2}$.

\section{Parallel shunt}

For the parallel shunt circuit, the inductance $L$ is determined by (96). When the modified capacitance $C_{L}$ is eliminated in terms of the squared effective EMCC $\kappa_{e}^{2}$ by (89) and (103), with the normalised modal stiffness expressed as $k_{r}=$ $m_{r} \omega_{r}^{2}$, the shunt inductance can be represented as

$$
L=\kappa_{e}^{2} \frac{m_{r}}{\bar{e}_{31}^{2}} .
$$

In this expression, the normalised modal mass $m_{r}$ may often be estimated quite accurately from the vibration form of the structure, while the squared effective EMCC $\kappa_{e}^{2}$ is determined by (72). The resulting plane stress-reduced piezoelectric coupling coefficient $\bar{e}_{31}^{2}$ is further specified for the particular piezoceramic patches through (28) and wiring by (56) and (65) for respectively SP-PW and OP-SW pair of patches.

Once the inductance $L$ has been determined, a pure $L$-shunt is constructed, as indicated in Figure 4(b) with $R=0$. Hereby, two new resonant frequencies $\omega_{-}^{2}$ and $\omega_{+}^{2}$ emerge around the original SC frequency $\omega_{r}^{2}$. Expressions for these two frequencies can be determined by inserting the expression for the inductance (96) into the characteristic equation (104) and considering the expressions for the residual mode corrected modal EMCCs (89) and (90), whereby the following quadratic equation in $\omega^{2}$ is obtained,

$$
\omega^{4}-\left(2+\kappa_{r}^{2}\right) \omega_{r}^{2} \omega^{2}+\omega_{r}^{4}=0 .
$$


Table 2. Tuning procedure based on the effective EMCC for parallel and series shunt circuits.

\begin{tabular}{ccc} 
Parameter & Parallel & Series \\
\hline$L$ & $\kappa_{e}^{2} \frac{m_{r}}{\bar{e}_{31}^{2}}$ & $\kappa_{e}^{2} \frac{m_{r}}{\left(1+\kappa_{e}^{2}\right)^{2} \bar{e}_{31}^{2}}$ \\
$R$ & $\kappa_{r}^{2} \frac{m_{r} \omega_{r}}{\bar{e}_{31}^{2}} \sqrt{\frac{1}{2 \kappa_{r}^{2}}}$ & $\kappa_{r}^{2} \frac{m_{r} \omega_{r}}{\bar{e}_{31}^{2}} \sqrt{\frac{2 \kappa_{r}^{2}}{\left(1+\kappa_{r}^{2}\right)^{3}}}$
\end{tabular}

Initial step:

Determine:

$$
\kappa_{e}^{2}=\frac{\hat{\omega}^{2}-\omega^{2}}{\omega} \quad, \quad L=\kappa_{e}^{2} \frac{m_{r}}{\bar{e}_{31}^{2}}
$$

$$
\omega_{+}, \omega_{-} \quad, \quad \kappa_{r}^{2}=\frac{\left(\omega_{+}-\omega_{-}\right)^{2}}{\omega_{+} \omega_{-}}
$$

The product and sum of the solutions $\omega_{+}^{2}$ and $\omega_{-}^{2}$ can be written as

$$
\omega_{+}^{2} \omega_{-}^{2}=\omega_{r}^{4} \quad, \quad \omega_{+}^{2}+\omega_{-}^{2}=\left(2+\kappa_{r}^{2}\right) \omega_{r}^{2}
$$

and elimination of $\omega_{r}^{2}$ between these relations gives the modal EMCC $\kappa_{r}^{2}$ as

$$
\kappa_{r}^{2}=\frac{\left(\omega_{+}-\omega_{-}\right)^{2}}{\omega_{+} \omega_{-}}
$$

The shunt resistance then follows from (95) as

$$
R=\kappa_{r}^{2} \frac{m_{r} \omega_{r}}{\bar{e}_{31}^{2}} \sqrt{\frac{1}{2 \kappa_{r}^{2}}}
$$

where $C_{r}$ has been eliminated by (90) and $k_{r}=\omega_{r}^{2} m_{r}$ has again been used. As for the inductance $L$ in (105), the resistance $R$ is determined by the normalised modal mass and the resulting plane stress-reduced piezoelectric coupling coefficient $\bar{e}_{31}^{2}$, while $\kappa_{r}^{2}$ should be used for $R$ instead of $\kappa_{e}^{2}$. The tuning formulas for the optimal parallel connected inductance and resistance based on the effective EMCC are summarised in the second column of Table 2.

\section{Series shunt}

For the series shunt circuit, the same approach is used. The inductance $L$ is calibrated by the expression in (101), where the assumption $\kappa_{L}^{2} \xi \ll 1$ yields the simplified expression

$$
L=\kappa_{e}^{2} \frac{m_{r}}{\left(1+\kappa_{e}^{2}\right)^{2} \bar{e}_{31}^{2}},
$$

which only contains the squared effective EMCC $\kappa_{e}^{2}$ from (103), the normalised modal mass $m_{r}$ and the squared resulting plane stress-reduced piezoelectric coupling coefficient $\bar{e}_{31}^{2}$, while $C_{L}$ is eliminated by (89), (103) and $k_{r}=\omega_{r}^{2} m_{r}$. Again, the pure $L$-shunt with $R=0$ in Figure $4(\mathrm{~b})$ is constructed and the two natural frequencies $\omega_{+}$and $\omega_{-}$are determined numerically or experimentally. The shunt resistance is given by the expression in (101b), in which the modal resistance $R_{r}$ from (100) is scaled by the apparent inductance ratio $L / L_{r}$, where the modal inductance in (100) alternatively can be written as

$$
L_{r}=\kappa_{r}^{2} \frac{m_{r}}{\left(1+\kappa_{r}^{2}\right)^{2} \bar{e}_{31}^{2}}
$$

when introducing (89), (103) and $k_{r}=\omega_{r}^{2} m_{r}$. Thus, the shunt resistance can be obtained by the expression

$$
R=\kappa_{r}^{2} \frac{m_{r} \omega_{r}}{\bar{e}_{31}^{2}} \sqrt{\frac{2 \kappa_{r}^{2}}{\left(1+\kappa_{r}^{2}\right)^{3}}}\left(\frac{L}{L_{r}}\right)^{2}
$$

which besides the normalised modal mass $m_{r}$, the resulting plane stress-reduced piezoelectric coupling coefficient $\bar{e}_{31}^{2}$ and the squared modal EMCC $\kappa_{r}^{2}$, also depends on the squared effective EMCC $\kappa_{e}^{2}$ through the ratio $L / L_{r}$. However, while the calibration of the inductance must be calibrated rather precisely because it governs the shunt frequency, the corresponding shunt resistance may subsequently be approximated by assuming $L_{r} / L \simeq 1$, as the shunt performance is less sensitive to deviations in the resistance. The approximated tuning formulas for the series connected resistance and the corresponding expression for the inductance are listed in the last column of Table 2.

For both the parallel and series shunt circuits, accurate calibration of the inductance and resistance can be achieved from the effective EMCC only, when the inertia correction is significantly smaller than the flexibility correction $1 /\left(\omega_{r}^{2} L_{r}^{\prime}\right)<<C_{r}^{\prime}$, whereby $\kappa_{r} \simeq \kappa_{e}$. This is often the case for the lower and well separated vibration modes, while the influence of the inertia correction may increase for the higher and closely spaced modes. It is beneficial to base the shunt tuning on the effective EMCC only, as one avoids the evaluation of an additional eigenvalue problem or experiments with the pure $L$-shunt, which can be difficult to produce due the inherent resistance in both synthetic and purely passive inductors.

\section{Error estimate}

The error arising from the use of either a single-mode representation $\kappa_{0}^{2}$ or the method with pure quasi-static corrections $\kappa_{r}^{2}$ for the shunt circuit calibration can for the parallel shunt be represented by the ratio $\kappa^{2} / \kappa_{e}^{2}$, which is unity when the residual mode correction is insignificant. For the series shunt, this ratio provides a good estimate of the error, although it is not entirely unity because of the truncations introduced for this shunt. Thus, the necessity of including both the flexibility and the inertia contributions from the non-resonant modes can be evaluated by comparing the different squared modal EMCCs: $\kappa_{0}^{2}, \kappa_{r}^{2}$ and $\kappa_{L}^{2}$ with the squared effective EMCC $\kappa_{e}^{2}$, as demonstrated in the following benchmark examples.

In the shunt tuning formulas in Table 2, the effect of having, respectively, an SP-PW or an OP-SW configured pair of piezoceramic patches is directly seen to be governed by the corresponding resulting plane stressreduced piezoelectric coupling coefficient $\bar{e}_{31}^{2}$. The SC and OC frequencies and thereby the squared effective EMCC will be the same for the structure with respectively a pair of patches in SP-PW and OP-SW configurations. Consequently, the squared modal EMCC $\kappa_{r}^{2}$ will as well be identical for the two configurations. Finally, since the normalised modal mass $m_{r}$ is also independent of the poling and wiring of the pair of piezoceramic pacthes, it is found that both the optimal inductance and resistance for the OP-SW pair of patches are four times as large as the corresponding values for the 
SP-PW patch configuration. This is because the resulting plane stress-reduced piezoelectric coupling coefficient for the SP-PW and OP-SW pairs of patches are respectively $2 e_{31}$ and $e_{31}$, see (56) and (65). Hence, as $\bar{e}_{31}$ is squared in the denominator of the tuning formulas in Table 2, a factor four occurs between the shunt tunings of the two respective pair of patches configurations. In Thomas et al. (2009) and Lossouarn et al. (2017), the same cantilever beam with a single pair of respectively OP-SW and SP-PW piezoceramic patches is analyzed. Considering the corresponding optimal shunt tunings based on experiments, it is approximately found that the OP-SW configured patches cause four times larger optimal inductance and resistance values compared to the SP-PW configuration.

\section{Benchmark Examples}

In this section, the balanced calibration procedure based on the modal and effective EMCC, respectively, are analyzed. It is noted that only one shunt tuning will be provided in the examples as the two methods give almost the same tuning values. The small deviations are only due to the approximation (103).

The implemented FE-model enables comparison with shunt tuning methods from benchmark examples in the literature concerning optimal $R L$-shunt tuning. The two examples of this section consider a cantilever beam, analyzed by both Thomas et al. $(2009,2012)$ and Ducarne et al. (2012), and a plate analyzed by Gardonio and Casagrande (2017). In both cases the structure is equipped with a single pair of piezoceramic patches, configured either as OPSW or SP-PW. The present examples apply the particular configurations from the benchmark cases, although the SPPW configuration is seemingly adequate, as it leads to four times smaller inductances and resistances than for OP-SW.

As experimental results are available for the cantilever beam example, it can be used to verify the established numerical model as well as assess the effect of using beam instead of plate elements for the modelling of plate-like beam structures. For the subsequent plate benchmark presented by Gardonio and Casagrande (2017), the influence of the non-resonant modes are investigated by a thorough parametric study. Therefore, this example constitutes a good opportunity to compare the benchmark results with the present calibration procedures, in which the presence of the non-resonant modes are accounted for explicitly.

\section{Cantilever beam}

The first example concerns the cantilever beam with a single pair of OP-SW piezoceramic patches, for which the $\mathrm{SC}$ and $\mathrm{OC}$ frequencies and the associated modal EMCC are determined both numerically and experimentally by Thomas et al. (2009). The optimal tuning of the electronic components for both $R$ - and $R L$-shunts is provided in Thomas etal. (2012), while the optimum design and placement of the piezoceramic patches are analyzed by Ducarne et al. (2012). The geometry of the cantilever beam can be seen in Figure 5. The single pair of ideally bonded OP piezoceramic patches (red color) are placed in series with a shunt circuit (blue color) with impedance $Z_{s h}(\omega)$. The beam is conductive and a conductive adhesive is used for the

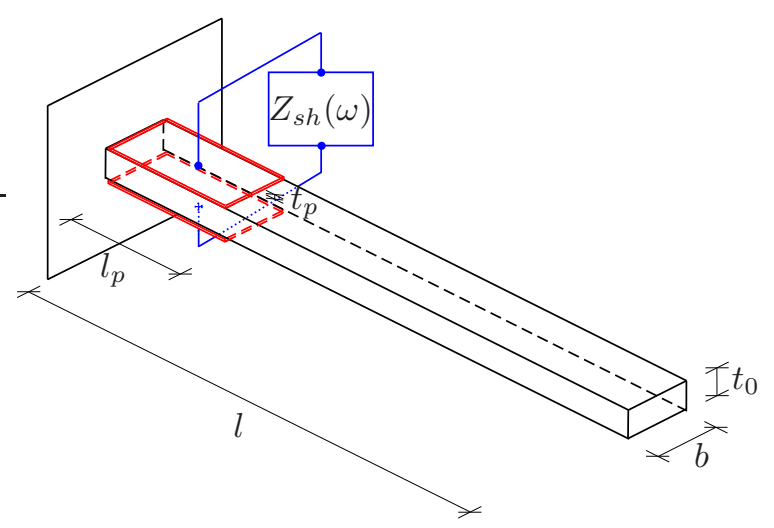

Figure 5. Geometry of cantilever beam, with one pair of OP, surface bonded and SW piezoceramic patches.

Table 3. Dimensions and material properties for cantilever beam and piezoceramic patches.

\begin{tabular}{lcccc} 
& \multicolumn{2}{c}{ Beam } & \multicolumn{2}{c}{ Piezo } \\
\hline Length $(\mathrm{mm})$ & $l$ & 170 & $l_{p}$ & 25 \\
Width $(\mathrm{mm})$ & $b$ & 20 & $b$ & 20 \\
Thickness $(\mathrm{mm})$ & $t_{0}$ & 2 & $t_{p}$ & 0.5 \\
& & & & \\
Density $\left(\mathrm{kg} / \mathrm{m}^{3}\right)$ & $\rho$ & 2800 & $\rho_{p}$ & 8500 \\
Young's Modulus $(\mathrm{GPa})$ & $E$ & 72 & $E_{p}$ & 66.7 \\
Poisson's ratio (-) & $\nu$ & 0 & $\nu_{p}$ & 0 \\
& & & & \\
Piezoelectric coefficient $\left(10^{-12} \mathrm{~m} / \mathrm{V}\right)$ & $d_{31}$ & -210 \\
Blocked dielectric coefficient $(\mathrm{F} / \mathrm{m})$ & $\epsilon_{33}^{\varepsilon_{p}}$ & $2068 \varepsilon_{0}$ \\
\hline
\end{tabular}

$\varepsilon_{0}=8.854 \times 10^{-12} \mathrm{~F} / \mathrm{m}$, see IEEE inc. (1988)

bonding of the piezoceramic patches, whereby the two inner electrodes of the patches are connected. The pair of patches is modelled as described in the first section, where the resulting capacitance and plane stress-reduced piezoelectric coupling coefficient can be determined by (65).

The dimensions and material properties of the beam and the piezoceramic patches are given in Table 3. A vanishing Poisson's ratio is assumed for both patch and beam, whereby the present results can be compared to the numerical results for the beam model in Thomas et al. (2009). In the original experimental design, an additional tip mass $m_{e x}=4.2 \mathrm{~g}$ is added for excitation purpose. This mass is therefore also added to the diagonal element of the mass matrix for the transverse dof of the center node at the free end of the beam. Finally, the pair of piezoceramic patches is placed with a horizontal offset of $x_{p}=0.5 \mathrm{~mm}$ relative to the fixed support.

The cantilever beam is discretized by $41 \times 2$ plate elements, while the piezoelectric patches are each represented by $5 \times 2$ patch elements. The corresponding beam model (Høgsberg and Krenk, 2015) with 41 Bernoulli beam elements and 5 (1D) patch elements is used for comparison. It corresponds to the model in Thomas et al. (2009) with 41 beam elements, with 5 elements containing the electromechanical coupling to the pair of patches and it reproduces the first two vibration modes with sufficient accuracy.

In order to verify the current electromechanical piezoplate model, the first two SC and OC frequencies are determined. They are summarized in Table 4, which also 
Table 4. SC and OC frequencies, modal EMCC and shunt circuit calibration for modes 1 and 2 of a cantilever beam, using a beam and a plate model.

\begin{tabular}{|c|c|c|c|c|c|c|}
\hline & $\begin{array}{l}\text { Model } \\
\text { Shunt }\end{array}$ & $f[\mathrm{~Hz}]$ & $\hat{f}[\mathrm{~Hz}]$ & $\kappa$ & $L[\mathrm{H}]$ & $R[\mathrm{k} \Omega$ \\
\hline & Plate & & & & & \\
\hline & Parallel ${ }^{*}$ & & & 018 & 991.0 & 1619 \\
\hline & Series* & 48.93 & 49.36 & 0.13 & 957.0 & 55.85 \\
\hline- & Parallel $^{0}$ & & & & 1156 & 1749 \\
\hline$\frac{0}{8}$ & Series $^{0}$ & 48.93 & 49.36 & 0.14 & 1110 & 70.00 \\
\hline$\sum^{0}$ & Beam & & & & & \\
\hline & Series* & 48.93 & 49.39 & 0.14 & 1021 & $61.6 \mathrm{C}$ \\
\hline & Series $^{1}$ & 48.96 & 49.42 & 0.14 & 1131 & 61.56 \\
\hline & Experim & & & & & \\
\hline & Series $^{2}$ & 51.64 & 52.17 & 0.14 & $1020^{3}$ & $58.6^{3}$ \\
\hline & Plate & & & & & \\
\hline & Parallel* & 3270 & 3403 & 014 & 21.26 & 226.2 \\
\hline & Series* & $53 \%$ & 340.3 & 0.14 & 20.45 & 8.702 \\
\hline & Parallel $^{0}$ & 3270 & & & 24.36 & 242.5 \\
\hline$\frac{0}{8}$ & Series $^{0}$ & 3. & .3 & 0.15 & 23.29 & 10.61 \\
\hline$\sum$ & Beam & & & & & \\
\hline & Series* & 337.1 & 340.7 & 0.15 & 21.84 & 9.63 \\
\hline & Series $^{1}$ & 337.1 & 340.7 & 0.15 & 23.81 & 9.37 \\
\hline & Experin & & & & & \\
\hline & Series $^{2}$ & 337.0 & 340.2 & 0.14 & $23.9^{3}$ & $8.65^{\circ}$ \\
\hline
\end{tabular}

* Present model with residual mode correction $\left(\kappa_{L}\right)$

${ }^{0}$ Present model without residual mode correction $\left(\kappa_{0}\right)$

${ }^{1}$ Tuning based on theory in Thomas et al. (2012) $\left(\kappa_{0}\right)$

${ }^{2}$ Experimental results from Thomas et al. (2012) $\left(\kappa_{e}\right)$

${ }^{3}$ Final values not measured Thomas et al. (2012)

contains the experimental and numerical frequencies for the first bending modes reported in Thomas et al. (2009).

The optimum series and parallel shunt tuning of the pair of piezoceramic patches, based on the present piezoplate model, is now determined with and without residual mode contribution. The results are shown in the first rows of Table 4 for modes 1 and 2 . The table also contains the optimal series shunt tuning with residual mode correction for the simple beam model. For comparison Table 4 also presents the optimal experimental shunt tuning (Thomas et al. , 2012) (superscript 2), the theoretical tuning based on the tuning formulas without correction (Thomas et al., 2012)(superscript 1) and numerical beam model described in Thomas et al. (2009). Finally, the modal EMCCs $\kappa$, in Table 4, are calculated respectively with (89) and without (87) residual mode correction, while $\kappa$ 's provided for the experiment are the effective EMCC (72).

The reported numerical results based on the tuning formulas in Thomas et al. (2012) are obtained by the numerical beam model described in Thomas et al. (2009). They are seen to be in good agreement with the frequencies determined by the beam model in Høgsberg and Krenk (2015). The reason for the slightly lower frequencies is mainly due to the omission of the patches bending contribution in the present model. Good agreement is also observed for the determined SC and OC frequencies for the plate model, where the small decrease of the OC frequency occurs because of plate effects at the supports, which is not
Table 5. The effective EMCC $\left(\kappa_{e}\right)$ and the ratio between this squared and the squared modal EMCC, with $\left(\kappa_{L}^{2}\right)$ and without $\left(\kappa_{0}^{2}\right)$ residual mode correction.

\begin{tabular}{ccccc} 
Mode & $\kappa_{e}$ & $\kappa_{0}^{2} / \kappa_{e}^{2}$ & $\left(\kappa_{L}^{2} / \kappa_{e}^{2}\right)^{1}$ & $\kappa_{L}^{2} / \kappa_{e}^{2}$ \\
\hline 1 & 0.1330 & 1.1661 & 1.0694 & 1.0000 \\
2 & 0.1405 & 1.1457 & 1.0682 & 1.0001 \\
\hline 1 & &
\end{tabular}

captured by the simpler beam model. The experimentally obtained SC and OC frequencies (Thomas et al., 2012) are for the first mode slightly larger, while the mode 2 frequencies are in very good agreement with the numerical results. Significant differences are found between the modal EMCCs $\kappa_{L}$ and $\kappa_{0}$ with- and without residual mode correction, respectively. Furthermore, the corrected modal EMCC $\kappa_{L}$ from the beam model is seen to deviate from the corresponding EMCC determined by the plate model, demonstrating that the influence from the residual modes depends on the model accuracy. The effective EMCC based on the experimental data is expected to correspond well with the modal EMCC $\kappa_{L}$. However, it is seen to be larger for the first vibration mode, due to differences between the associated SC and OC frequencies. For the second vibration mode, the SC and OC frequencies are in better agreement, whereby the effective EMCC determined by (72) is more accurately represented by $\kappa_{L}$.

Comparing the values of the shunt resistances and inductances in Table 4 shows that the spill-over from the non-resonant residual vibration modes has a significant effect on the calibration. For both vibration modes, the relative deviation is approximately $15 \%$ on the shunt inductance between the calibration with and without residual mode correction for both series and parallel shunts. Because of this deviation in inductance, substantial changes in the corresponding resistance are also observed. It is noted that the final electronic components based on experiments are not measured, but merely stated in Thomas et al. (2012) to be close to the theoretical values. This indicates that a precise shunt tuning has not been obtained prior to the experiments.

The effective EMCC's for the first two beam vibration modes are provided in the first column of Table 5. Further, the ratio between the squared modal EMCCs and the squared effective EMCCs are compared in the second to last column of Table 5. It is found that the ratio between $\kappa_{0}^{2}$ (without correction) and the squared effective EMCC $\kappa_{e}^{2}$ exactly accounts for the observed error in the inductance for the parallel shunt, while it accounts approximately for the error for the series shunt, see Table 5. It follows from the last two columns of Table 5 that the residual mode corrected squared modal EMCC $\kappa_{L}^{2}$ almost exactly recovers the squared effective EMCC $\kappa_{e}^{2}$. When $\kappa_{L}^{2}$ is determined by the beam model, the deviation is around $7 \%$, while it exactly matches $\kappa_{e}^{2}$ for the plate model. This shows that the accuracy of the numerical model also influences the residual mode correction and thus the subsequent shunt tuning.

The effect of including the residual mode contributions in the shunt tuning is now illustrated by frequency response plots in Figure 6, for the cantilever beam exposed to a harmonic tip load with excitation frequencies around the first and second resonant frequencies. The response is determined 
(a)

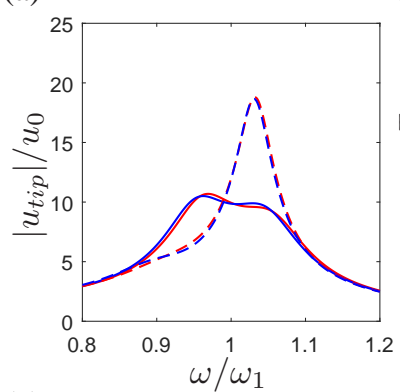

(c)

(b)

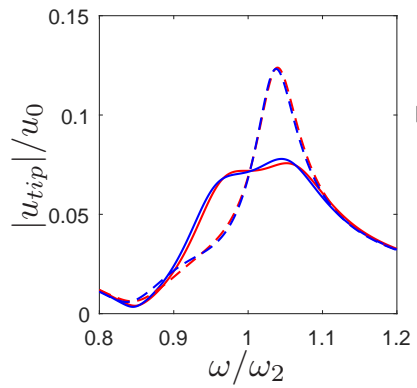

(d)
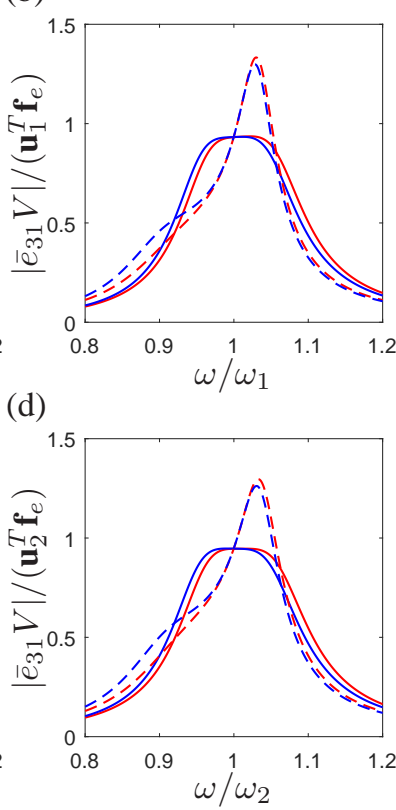

Figure 6. Frequency response around modes 1 and 2 of a cantilever beam exposed to a harmonic tip load; $(a, c)$ tip displacement, (b,d) voltage response. Blue lines indicate parallel and red lines series shunts, tuned respectively with (solid) and without (dashed) residual mode correction.

by solving the full system of equations for the parallel (75) and series (77) shunts with optimal components in Table 4.

It can be seen from Figure 6 that the calibrations without residual mode correction (dashed lines) cause significantly larger maximum amplification of both the tip displacement and voltage, compared to the tuning including the contribution from the non-resonant modes (solid lines). In particular, for the voltage amplification, an almost flat plateau around both resonant modes is seen for the calibration with the residual mode correction. The small deviations from a completely flat plateau are caused by the inclusion of structural damping in the model and from the fact that we do not have an ideal modal load, which is not accounted for by the calibration method. The structural damping is represented by Rayleigh damping with the damping ratios $\zeta_{1}=0.34 \%$ and $\zeta_{2}=0.28 \%$ for the first two vibration modes (Thomas et al. , 2009). The damping introduced by the shunted piezoceramic patches can be assessed either directly by the obtained damping ratios or from the reduction in vibration amplitude, as summarized in Table 6. The damping ratios are determined by solving the full complex eigenvalue problems for the parallel (75) and series (77) shunt circuits. The table contains the two damping ratios for each mode, associated with the two resonant frequencies emerging around the original resonant frequency when introducing the resonant shunt circuit. It is noted that the two damping ratios in Table 6 are ordered according to the magnitude of their associated natural frequencies. The amplitude reduction is obtained as the logarithm to the ratio of the maximum amplification of the structure with SC patch electrodes to that with optimally shunted pair of
Table 6. Damping ratios and amplitude reductions of cantilever beam with optimally calibrated parallel and series shunted piezoceramic patches, for resonant modes 1 and 2.

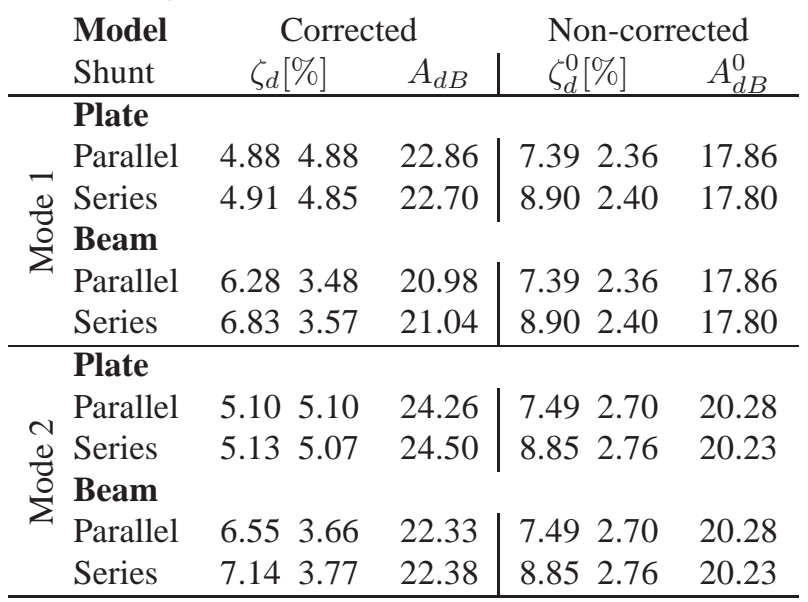

${ }^{0}$ Without residual mode correction

piezoceramic patches

$$
A_{d B}=20 \log \left(A_{S C} / A_{o p t}\right)
$$

It can be seen from the attained damping ratios and amplitude reductions in Table 6, that the inclusion of the nonresonant modes significantly improves the shunt calibration. The amplitude reduction is found to decrease about $35 \%$ from the best to the worst shunt calibration. It can also be seen that equal modal damping of the emerging resonant modes is obtained exactly for the parallel shunt with residual mode correction, while almost insignificant deviations occur for the corresponding series shunt, due to approximations made to simplify the tuning formulas. These results illustrate the potential improvement in common calibration methods, when taking into account the influence from non-resonant vibration modes. However, small discrepancies occur when the simplified beam model is used to obtain the desired level of damping or vibration mitigation. This illustrates the importance of having a sufficiently accurate numerical model for the dynamic structure, in order to obtain a precise shunt tuning. For the beam based shunt tuning without residual mode contribution, the damping ratios and amplitude reductions are identical to those obtained from the plate model. This is because the more substantial residual mode correction in the plate model is now omitted, while the corresponding vibration modes are almost identical.

\section{Simply supported plate}

The second example concerns a simply supported plate analyzed by Gardonio and Casagrande (2017), with a single SP-PW shunted pair of piezoceramic patches placed with a small offset of $\left(1 / 28 l_{x},-1 / 28 l_{y}\right)$ to the plate center. The plate geometry and the geometry and position of the pair of piezoceramic patches can be seen in Figure 7, while the dimensions and material properties are provided in Table 7. It is noted that the plate is conductive and that a conductive adhesive is used for the bonding of the piezoceramic patches, whereby the inner electrodes are connected. The structural damping is assumed to be $\zeta_{s}=0.2 \%$, and side lengths of the pair of patches are $1 / 5$ of the corresponding lengths of the plate $\left(l_{p x, p y}=l_{x, y} / 5\right)$. As the piezoceramic patches in 


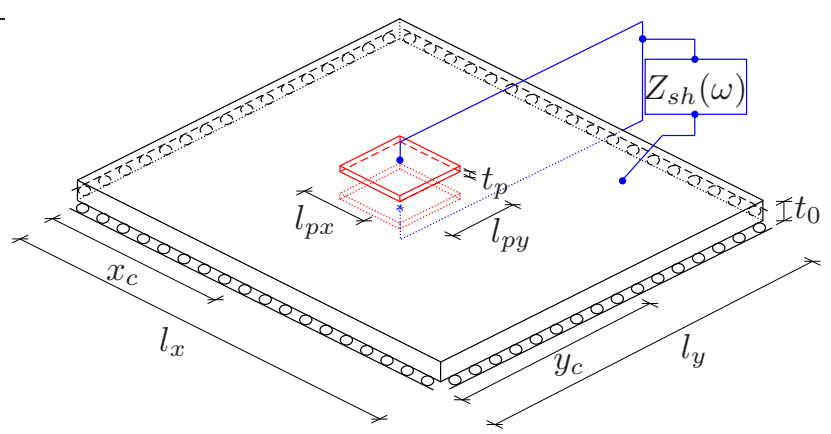

Figure 7. Geometry of a simply supported plate with one pair of SP-PW piezoceramic patches.

Table 7. Dimensions and material properties for the plate and piezoceramic patches.

\begin{tabular}{|c|c|c|c|c|}
\hline & \multicolumn{2}{|c|}{ Plate } & \multicolumn{2}{|c|}{ Piezo } \\
\hline Length (mm) & & 414 & $l_{p x}$ & 82.8 \\
\hline Width (mm) & $l_{y}$ & 314 & $l_{p y}$ & 62.8 \\
\hline Thickness (mm) & $t_{0}$ & 1 & $t_{p}$ & 0.5 \\
\hline \multicolumn{3}{|c|}{ Center patch along $\mathrm{X}$-axis $(\mathrm{mm})$} & $x_{c}$ & $\frac{13}{28} l_{x}$ \\
\hline \multicolumn{3}{|c|}{ Center patch along $\mathrm{y}$-axis (mm) } & $y_{c}$ & $\frac{15}{28} l_{y}$ \\
\hline Density $\left(\mathrm{kg} / \mathrm{m}^{3}\right)$ & $\rho$ & 2700 & $\rho_{p}$ & 7600 \\
\hline Young's Modulus (GPa) & $E$ & 70 & $E_{p}$ & 50 \\
\hline Poisson's ratio (-) & $\nu$ & 0.33 & $\nu_{p}$ & 0.35 \\
\hline \multirow{2}{*}{\multicolumn{3}{|c|}{$\begin{array}{l}\text { Piezoelectric coefficient }\left(10^{-12} \mathrm{~m} / \mathrm{V}\right) \\
\text { Free dielectric coefficient }\left(10^{-9} \mathrm{~F} / \mathrm{m}\right)\end{array}$}} & $d_{31}$ & -150 \\
\hline & & & $\epsilon_{33}^{\sigma_{p}}$ & 29.2 \\
\hline
\end{tabular}

the present example are relatively thick and placed far from the boundary simple supports, the omission of the patches bending stiffness and transverse inertia becomes significant. Thus, the additional mass from the pair of piezoceramic patches is lumped and included at the relevant dofs in the system mass matrix. Hereby, the present results become comparable with those in Gardonio and Casagrande (2017).

In Gardonio and Casagrande (2017), the optimal calibration of the parallel $R L$-shunts electronic components is determined for a particular load case, described as a rain on the roof load scenario. The solution is evaluated using a mode shape expansion, with a gradually increasing number of modes. The solution is seemingly converged when 150 modes are included. The bottom row of Table 8 provides the fundamental frequencies of the plate, both with and without SC patches, and the optimal tuning components from Gardonio and Casagrande (2017). The two top rows of the table summarize the corresponding results obtained by the present balanced calibration procedure, with and without residual mode correction. The present FE-model consists of $28 \times 28$ plate elements and $6 \times 6$ patch elements, whereby the relative errors on the first eight SC frequencies of the coupled piezo-plate structure is below one percent, as demonstrated by the convergence curves in Figure 8 with reference values obtained by a fine mesh with $112 \times 112$ plate and $24 \times 24$ patch elements. Furthermore, the straight lines in the logarithmic scale of Figure 8 indicate quadratic convergence. The SP-PW pair of piezoceramic patches is modelled according to the piezo-plate coupling subsection
Table 8. Fundamental frequencies of simply supported plate with $(f)$ and without $\left(f_{0}\right)$ short-circuit piezoceramic patches, modal EMCC and optimal shunt tuning.

\begin{tabular}{lcccrr} 
Shunt & $f_{0}[\mathrm{~Hz}]$ & $f[\mathrm{~Hz}]$ & $\kappa$ & $L[\mathrm{H}]$ & $R[\mathrm{k} \Omega]$ \\
\hline Parallel $^{*}$ & \multirow{2}{*}{39.05} & \multirow{2}{*}{35.73} & 0.070 & 33.87 & 76.89 \\
Parallel $^{0}$ & & & 0.074 & 36.92 & 80.31 \\
Parallel $^{1}$ & $\simeq 39.1$ & $\simeq 37.2$ & - & $\simeq 34$ & $\simeq 160$ \\
\hline
\end{tabular}

* With residual mode correction $\left(\kappa_{L}\right)$

${ }^{0}$ Without residual mode correction $\left(\kappa_{0}\right)$

${ }^{1}$ Results from Gardonio and Casagrande (2017)

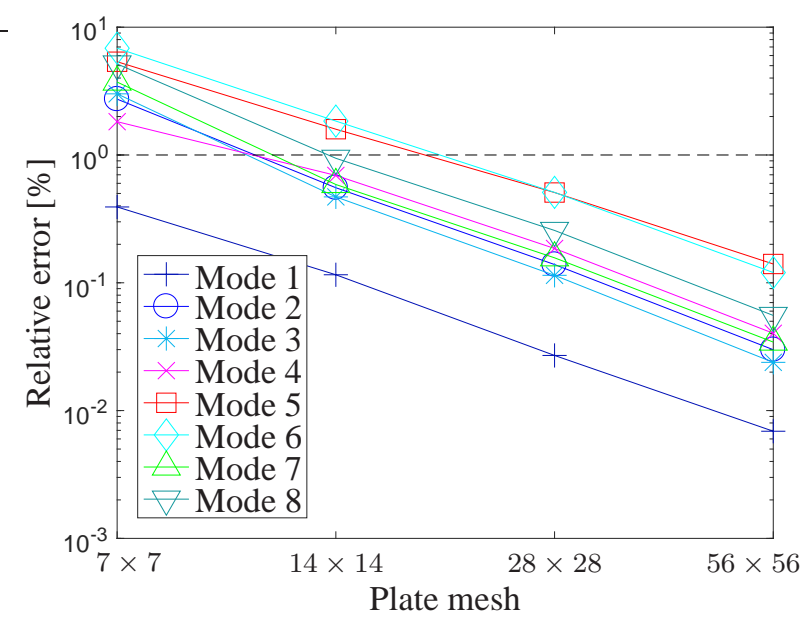

Figure 8. Relative error on first eight SC frequencies of piezoplate structure (reference, refined mesh $112 \times 112$ ).

in the first section, with the resulting capacitance and plane stress-reduced piezoelectric coupling coefficient determined from (56). The blocked dielectric constant

$$
\epsilon_{33}^{\varepsilon_{p}}=\epsilon_{33}^{\sigma_{p}}-2 d_{31} \bar{e}_{31} .
$$

is determined from the free dielectric constant $\epsilon_{33}^{\sigma_{p}}$ in Table 7.

Good agreement between the base plate frequencies $\left(f_{0}\right)$ can be observed in Table 8, while the SC frequency of the piezo-plate structure $(f)$ is seen to be lower using the present FE-model. This discrepancy occurs because of the omission of the patches bending stiffness. Therefore, the presently determined SC frequencies will all be lower than actual frequencies (Gardonio and Casagrande, 2017). The present tuning of the inductance $L$ with residual mode correction is seen to be in good agreement with the tuning obtained by Gardonio and Casagrande (2017), while a significant deviation can be seen for the shunt resistance $R$. This agrees well with the fact that the present tuning formula for the inductance is identical to that in Gardonio and Casagrande (2017), while the present resistance is $\sqrt{2}$ smaller than in the previous study. The slightly smaller inductance is due to the differences in the SC frequencies, while the deviation on the resistance is dominated by the different tuning formulas based on free vibration properties in the present procedure and a specific forcing in Gardonio and Casagrande (2017). In Table 8 the calibration without residual mode contribution $\left(L_{0}, R_{0}\right)$ deviates about $9 \%$ for the inductance and $4.5 \%$ for the resistance, which is slightly less than in the previous beam example. This is because the patches in the present 
(a)

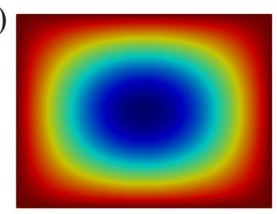

(b)

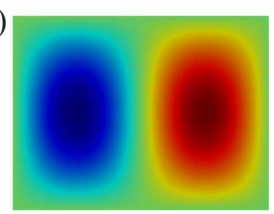

(c)

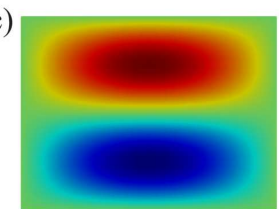

(d)

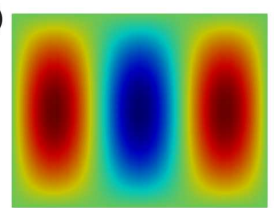

(e)

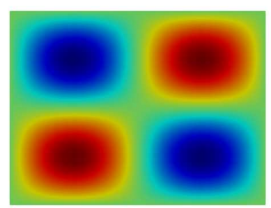

(f)

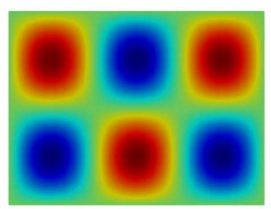

(g)

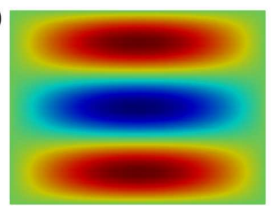

(h)

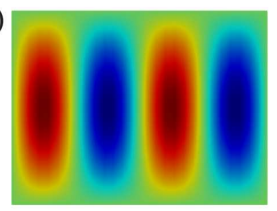

(i)

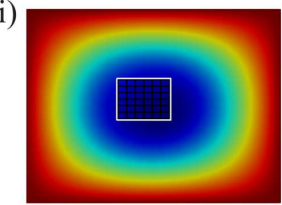

(j)

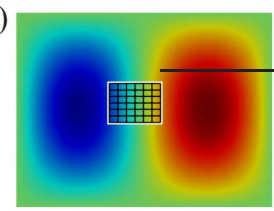

(k)

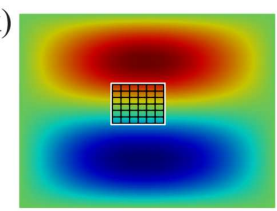

(1)

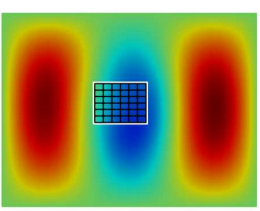

(m)

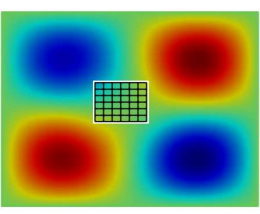

(n)

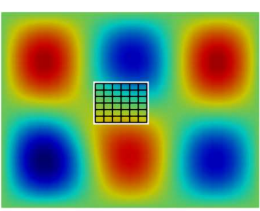

(o)

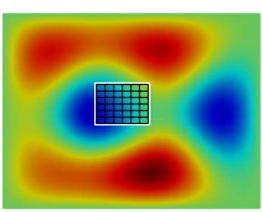

(p)

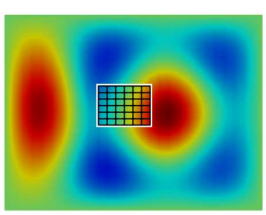

Figure 9. Plate modes 1-8 for a bare plate $(\mathrm{a}-\mathrm{h})$ and plate with a SC pair of piezoceramic patches (i-p).

plate example have reduced authority, whereas the larger capacitance of the present SP-PW patches configuration is equalled by the correspondingly larger plane stress-reduced piezoelectric coupling coefficient, see (56) and (65).

The magnitude of structural authority by the piezoceramic patches is determined by the relation between stiffness, mass and dimensions for the host structure and the patches in SC conditions. In the present case the low authority is caused by the small patch to plate area ratio, whereby the contribution from non-resonant modes becomes less pronounced than in the previous beam example. However, as it will be

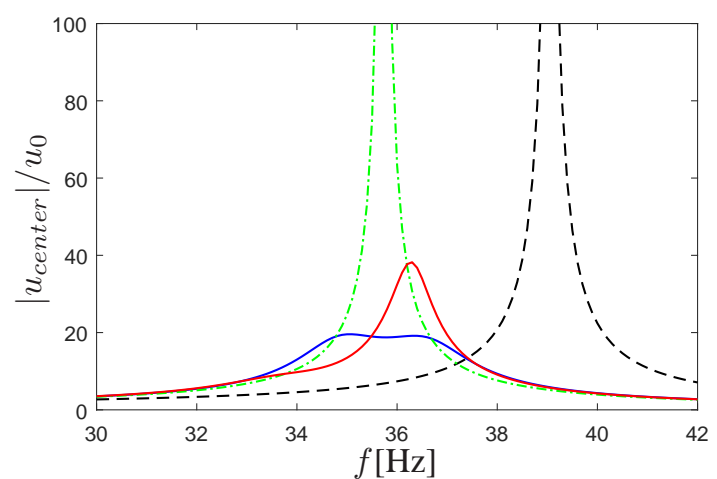

Figure 10. Frequency response around mode 1 for a plate (dashed black line) with a SC (green dot-dashed line) and parallel shunted pair of patches, optimally tuned with (blue line) and without (red line) residual mode correction.

shown subsequently, the low structural authority causes the shunt performance to be more sensitive to deviations from the optimum shunt tuning. The low structural authority implies reduced effective EMCCs, as the separation of the $\mathrm{SC}$ and $\mathrm{OC}$ frequencies in (72) is governed by the term $\left(\bar{e}_{31}^{2} / \bar{C}_{p}^{\varepsilon_{p}}\right) \mathbf{w} \mathbf{w}^{T}$ in (71). A significant effective EMCC and thereby large damping is attainable in the present plate example, when the pair of patches is placed optimally with respect to the deformation pattern of the concerned vibration mode. Figure 9 shows the first eight vibration modes of the base plate (a-h) and the plate with the SC patches (i-p). It is seen that the pair of patches is placed optimally for the first vibration mode $(\mathrm{a}, \mathrm{i})$, while the authority is reduced for the second mode $(b, j)$. To realize a desired level of damping by an indirectly placed pair of patches, requires either larger patch dimensions or improved electromechanical coupling, in which case the influence from residual modes will increase as well. Thus, it is important in these cases to include the influence from residual vibration modes in the shunt calibration. This is illustrated in Figure 10, which shows the dynamic amplification curve for harmonic distributed transverse load with constant spatial intensity. The figure shows the resonance peaks for the base plate (black dashed) and the plate with the SC pair of patches (green dasheddotted), and the curves obtained by shunt with (blue) and without residual mode correction (red). It is seen that the dynamic amplification is doubled, when neglecting the residual mode correction compared to the calibration with correction. In the beam example, a factor of two can also be observed between the dynamic amplifications associated with the shunt tunings with and without residual mode correction, see Figure 6(a). However, in the beam example the relative deviation on the shunt tuning is significantly larger than in the plate example. This indicates that the shunt tuning is more sensitive to deviations, when the piezoceramic patches have less structural authority. It should also be noted that the frequency response plots are produced by a dynamic point- and distributed load, respectively, in the beam and plate examples, which as well influence the responses.

Optimum calibration for the parallel shunt circuit with and without residual mode correction is now determined for the first eight vibration modes with the corresponding 
Table 9. Optimum parallel shunt tuning and attained damping ratios, with (w) and without (wo) residual mode correction, for the first eight vibration modes of simply supported plate.

\begin{tabular}{|c|c|c|c|c|c|c|c|}
\hline \multicolumn{2}{|c|}{ Mode } & \multirow{3}{*}{$\frac{f[\mathrm{~Hz}]}{35.73}$} & \multirow{2}{*}{$\frac{\kappa}{0.070}$} & \multirow{2}{*}{$\frac{L[\mathrm{H}]}{33.87}$} & \multirow{2}{*}{$\frac{\mathrm{R}[\mathrm{k} \Omega]}{76.89}$} & \multicolumn{2}{|c|}{$\zeta[\%]$} \\
\hline \multirow{2}{*}{1} & $\mathrm{~W}$ & & & & & 2.57 & 2.57 \\
\hline & wo & & 0.073 & 36.92 & 80.31 & 3.95 & 1.19 \\
\hline \multirow{2}{*}{2} & w & \multirow{2}{*}{80.03} & 0.016 & 6.740 & 152.9 & 0.66 & 0.66 \\
\hline & wo & & 0.016 & 7.358 & 160.3 & 1.08 & 0.23 \\
\hline \multirow{2}{*}{3} & w & \multirow{2}{*}{111.0} & 0.017 & 3.488 & 99.29 & 0.71 & 0.71 \\
\hline & wo & & 0.018 & 3.827 & 104.9 & 1.18 & 0.23 \\
\hline \multirow{2}{*}{4} & w & \multirow{2}{*}{153.4} & 0.074 & 1.840 & 16.74 & 2.72 & 2.71 \\
\hline & wo & & 0.078 & 2.003 & 17.59 & 1.31 & 0.21 \\
\hline \multirow{2}{*}{5} & w & \multirow{2}{*}{163.1} & 0.007 & 1.698 & 153.5 & 0.33 & 0.33 \\
\hline & wo & & 0.007 & 1.772 & 185.1 & 0.37 & 0.21 \\
\hline \multirow{2}{*}{6} & w & \multirow{2}{*}{227.8} & 0.007 & 0.801 & 90.90 & 0.36 & 0.35 \\
\hline & wo & & 0.008 & 0.908 & 116.8 & 0.50 & 0.20 \\
\hline \multirow{2}{*}{7} & W & \multirow{2}{*}{239.9} & 0.070 & 0.756 & 11.34 & 2.55 & 2.55 \\
\hline & wo & & 0.073 & 0.819 & 11.99 & 1.24 & 0.21 \\
\hline \multirow{2}{*}{8} & W & \multirow{2}{*}{247.3} & 0.005 & 0.772 & 107.8 & 0.27 & 0.27 \\
\hline & wo & & 0.005 & 0.771 & 169.8 & 0.23 & 0.24 \\
\hline & $W$ & & & & & & \\
\hline & wo & Wi & & d & tert & & \\
\hline
\end{tabular}

mode shapes shown in Figure 9(i-p) for SC patch electrodes. The results are summarized in Table 9, where the provided modal EMCCs are $\kappa=\kappa_{L}$ in (89) with residual mode correction and $\kappa=\kappa_{0}$ in (87) without correction. The last columns of the table show the two damping ratios for the targeted vibration mode, determined from the full complex eigenvalue problem in (75).

The results in Table 9 indicate that considerable damping is realized only for modes 1,4 and 7 . This is due to the low structural authority of the pair of piezoceramic patches and its indirect location with respect to the deformation form of the five remaining vibration modes in Figure 9. Some of the vibration modes in Figure 9 are very sensitive to the actuation by the pair of patches. For example, in modes $5(\mathrm{e}, \mathrm{m})$ and $6(\mathrm{f}, \mathrm{n})$ the symmetry in the pattern is slightly altered by the presence of the patch, while for modes $7(\mathrm{~g}, \mathrm{o})$ and $8(\mathrm{~h}, \mathrm{p})$ the shape is more drastically changed. As it was observed for the damping of the first vibration modes, the deviation on the shunt calibration, when neglecting the residual modes, leads to a significant detuning and thereby smaller minimum damping ratios of the first eight vibration modes, see Table 9. There also seems to be a connection between the magnitude of the modal EMCC and the deviations observed for shunt tuning without residual mode correction. This supports the previous conclusion that the effect of the residual mode correction is governed by the apparent structural authority of the piezoceramic patches.

The influence of using the flexibility and flexibilityinertia corrections, respectively, for the shunt tuning can be analyzed by looking at the relative errors on the squared modal EMCC, without $\kappa_{0}^{2}$ and with flexibility $\kappa_{r}^{2}$ and flexibility-inertia $\kappa_{L}^{2}$ residual mode corrections, in respect to the squared effective EMCC $\kappa_{e}^{2}$, provided in Table 10. It follows from Table 10 that the squared effective EMCC
Table 10. The squared effective EMCC and the ratio between this and the squared modal EMCC with flexibility, flexibility-inertia and no residual mode correction.

\begin{tabular}{ccccc} 
Mode & $\kappa_{e}$ & $\kappa_{0}^{2} / \kappa_{e}^{2}$ & $\kappa_{r}^{2} / \kappa_{e}^{2}$ & $\kappa_{L}^{2} / \kappa_{e}^{2}$ \\
\hline 1 & 0.0699 & 1.0899 & 1.0007 & 1.0000 \\
2 & 0.0156 & 1.0917 & 0.9976 & 1.0000 \\
3 & 0.0172 & 1.0974 & 1.0029 & 1.0000 \\
4 & 0.0744 & 1.0891 & 1.0006 & 1.0001 \\
5 & 0.0068 & 1.0442 & 0.9540 & 1.0000 \\
6 & 0.0074 & 1.1343 & 1.0364 & 1.0000 \\
7 & 0.0699 & 1.0826 & 0.9939 & 1.0002 \\
8 & 0.0050 & 0.9985 & 0.9123 & 1.0000 \\
\hline
\end{tabular}

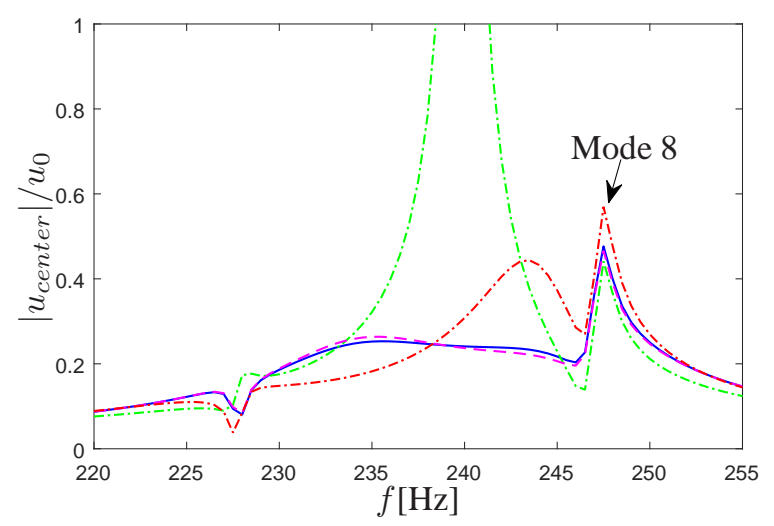

Figure 11. Frequency response around the seventh resonant frequency of a plate with pair of piezoceramic patches; SC (green dot-dashed line) and shunted optimally with flexibility-inertia (blue line), flexibility (dashed magenta line) and without (dotted-dashed red line) residual mode corrections.

$\left(\kappa_{e}^{2}\right)$ is determined very accurately by the squared modal EMCC $\left(\kappa_{L}^{2}\right)$ with residual mode correction. Also, the squared modal EMCC with pure flexibility correction $\left(\kappa_{r}^{2}\right)$ recovers the effective values rather precisely, in particular for the first four modes. For modes 5-8 the importance of using the more accurate flexibility-inertia corrections increases, especially for modes 5,6 and 8 , where the errors on the squared modal EMCCs $\left(\kappa_{r}^{2}\right)$ are significant. The latter may be due to the indirect placement of the pair of patches with respect to these modes. Generally, the importance of using the more substantial flexibility-inertia corrections for the non-resonant modes increases for resonant shunt damping of higher and closely spaced vibration modes with indirectly placed patches. The ratio between the squared modal and effective EMCC seems to correlate with relative errors observed for the (parallel) shunt inductance. It is for instance noted in Table 10 that $\kappa_{0}^{2} / \kappa_{e}^{2}$ indicates a 9\% relative error on the inductance, while for the first vibration mode the same order of error has been observed between $L$ and $L_{0}$. The error associated with the pure flexibility correction for the non-resonant modes observed for mode $5-8$ is now analyzed by considering the frequency response to a harmonic uniformly distributed load around the seventh resonant frequency shown in Figure 11.

It can be seen from Figure 11 that the calibration without residual mode correction (red dotted-dashed) causes significantly larger maximum amplifications compared to the tuning with flexibility-inertia residual mode correction (blue 
solid). The tuning with pure flexibility correction (magenta dashed) yields a small off-calibration, which shows that it may be important to use the precise flexibility-inertia compensation for the dynamic residual mode effects. In this example larger levels of attainable damping could have been achieved for the five lightly damped modes $(2,3,5$, 6 and 8 ) by either placing the pair of piezoceramic patches more appropriately, according to the deformation pattern of the respective modes, or by applying several interconnected pairs of patchess. The same position with larger patches or lower resulting capacitance could also have been realized, whereby the effects of including the non-resonant modes in the calibration would have been more pronounced. However, the design, optimisation and application of several pairs of patches is outside the main scope of the present work.

\section{Conclusions and future work}

An FE-model for coupled piezo-plate structures was established, by considering the constitutive relations and dynamic equilibrium equations of the plate, described by Kirchhoff theory, in which the patch was represented by a plane-stress assumption and its electromechanical interaction. The coupling between the plate and a pair of either SP-PW or OP-SW piezoceramic patches was considered through the transformation of the electric forcing from the two patches to the equivalent moment loads on the plate. The wiring and poling are subsequently implemented by considering the relations between the patch electrodes potentials and the voltage over the connected shunt. This reduces the number of electric equations to one, by the introduction of a resulting capacitance and a plane stress-reduced piezoelectric coupling coefficient. Hereby, the voltage across the shunt becomes the governing electric variable in the equations of motion.

A recently proposed balanced calibration procedure for $R L$-shunted piezoelectric transducers, with quasi-dynamic residual mode correction, is implemented for the shunted pair of piezoceramic patches bonded symmetrically to the plate. This calibration procedure enables precise and effective calibration of the electronic shunt components, as demonstrated by two benchmark examples concerning beam and plate vibrations.

A new calibration procedure based on the effective EMCC has also been proposed and used in the two benchmark examples. The method was derived from a new modal EMCC corrected by the spill-over from the non-resonant modes. In the benchmark examples, it is illustrated that this modal EMCC precisely retains the effective EMCC, hereby providing an explicit connection between the SC and OC frequencies of the coupled structure and the modal shunt tuning based on the numerical FE-model. It is further shown that the ratio between the squared modal EMCC with and without residual mode correction, and the corresponding squared effective EMCC provides the deviation in the corresponding shunt tuning. A simple format of the tuning formulas was obtained for the new calibration procedure. It showed that the difference in the shunt calibration of respectively an SP-PW or OP-SW pair of patches is simply determined by the reciprocal value of the squared resulting plane stress-reduced piezoelectric coupling coefficient. In the present case, this corresponds to four times higher shunt tuning values for the OP-SW pair of patches for a given level of attainable damping. Thus, it is often preferable to use this SP-PW configuration, as it is notoriously difficult to achieve the large inductances associated with damping of low-frequency structural vibrations.

The considered benchmark examples represent a cantilever beam and a simply supported plate, both equipped with a single pair of shunted piezoceramic patches configured in OP-SW and SP-PW, respectively. For the cantilever beam, optimum shunt tuning for vibration suppression of the first two resonant modes was determined and compared to former calibration results in the literature (Thomas et al., 2012). It was found that the omission of the residual mode correction leads to significant deviations on the shunt circuit electronic components. The latter is directly related to the ratio between the modal EMCC with and without residual mode correction. The deviation on the shunt tuning when neglecting the non-resonant modes leads to a further reduction in attainable damping. This was demonstrated directly by the damping ratios and by the amplitude reduction from frequency response curves for a particular harmonic load.

For the second benchmark example with a simply supported plate and a single pair of SP-PW piezoceramic patches, the effect of the non-resonant modes on the shunt tuning was found to be less pronounced. This is due to a smaller structural authority by the piezoceramic patches. However, notable influence by the residual mode correction is still observed on the frequency response curves and the damping ratios, verifying that the residual mode correction may be important in shunt tuning. It was found for the lower vibration modes that the residual mode corrections are sufficiently determined by the pure flexibility contribution, while the importance of using the flexibilityinertia corrections increases for higher and closely spaced vibration modes. This was illustrated by the frequency response curves at the first and the seventh vibration modes and the evaluation of the modal EMCC, both with flexibility and with flexibility-inertia residual mode corrections. At last, it was shown that the deviation on the shunt inductances can be determined by the ratio between the squared modal EMCC with and without residual mode correction and the corresponding squared effective EMCC. It was here demonstrated that the newly proposed residual mode corrected modal EMCC precisely retains the effective EMCC, which suggests that this coefficient should be used in shunt calibration based on modal analysis.

It is worth mentioning that the two proposed calibration methods give approximately the same shunt tuning as the corrected modal EMCC precisely retains the effective EMCC. The choice of method thus depends on whether the tuning is based on experiments or a numerical model and the associated computational costs. For the first balanced modal calibration procedure, the SC eigenvalue problem of the electromechanical structure has to be solved and a modified stiffness matrix has to be inverted in order to determine the residual mode correction terms. For the calibration based on the effective EMCC, both the SC and OC and an additional third eigenvalue problem, after determination of the optimal inductance, have to be evaluated. However, in many cases the inversion of the modified stiffness matrix will be associated 
with significant computational costs, thus the new calibration procedure, based on the effective EMCC, will in many cases be superior, also in regards of experimentally based calibration.

In future works, the proposed calibration procedures will be implemented in more sophisticated numerical models, allowing for even more accurate modelling of an experimental set-up or an industrial application. It is expected that the new procedure with the corrected modal EMCC will result in good agreement between optimum numerical and experimental based shunt tuning.

\section{Acknowledgements}

This research has been supported by the Danish Council for Independent Research via the project 'Resonant Piezoelectric Shunt Damping of Structures'.

\section{References}

Benjeddou A (2000) Advances in piezoelectric finite element modeling of adaptive structural elements: a survey. Computers and Structures, 76:347-363.

Benjeddou A (2002) Modelling of piezoelectric adaptive beam, plate and shell structures: some developments and results. Proceedings of the sixth International Conference on Computational Structures Technology Available at: http://www.ctresources.info/ccp/download/ccp.5245.pdf

Benjeddou A (2014) Modal effective electromechanical coupling approximate evaluations and simplified analyses: Numerical and experimental assessments. Acta Mechanica 225:27212742.

Berardengo M, Thomas O, Giraud-Audine C, Manzoni S (2016) Improved resistive shunt by means of negative capacitance: new circuit, performances and multi-mode control. Smart Materials and Structures, 25:075033.

Caruso G (2001) A critical analysis of electric shunt circuits employed in piezoelectric passive vibration damping. Smart Materials and Structures, 10:1059-1068.

Chevallier G, Ghorbel S, Benjeddou A (2009) Piezoceramic shunted damping concept: testing, modelling and correlation. Mechanics and Industry, 5:397-411.

Delperro T, Bergamini AE, Ermanni P (2012) Identification of electromechanical parameters in piezoelectric shunt damping and loss factor prediction, Journal of Intelligent Material Systems and Structures 24:287-298.

de Marneffe B and Preumont A (2008) Vibration damping with negative capacitance shunts: theory and experiment. Smart Materials And Structures 17:035015

Ducarne J, Thomas O, Deü J-F (2010) Structural vibration reduction by switch shunting of piezoelectric elements: modeling and optimization. Journal of Intelligent Material Systems and Structures 21:797-816.

Ducarne J, Thomas O, Deü J-F (2012) Placement and dimension optimization of shunted piezoelectric patches for vibration reduction. Journal of Sound and Vibration 331:3286-3303.

Forward RL (1979), Electronic damping of vibrations in optical structures. Applied Optics 18:690-697.

Gardonio P and Casagrande D (2017) Shunted piezoelectric patch vibration absorber on two-dimensional thin structure: Tuning considerations. Journal of Sound and Vibration 395:26-47.
Hagood NW and von Flotow A (1991) Damping of structural vibrations with piezoelectric materials and passive electrical networks. Journal of Sound and Vibration 146:243-268.

Høgsberg J and Krenk S (2012) Balanced calibration of resonant shunt circuits for piezoelectric vibration control. Journal of Intelligent Material Systems and Structures 23:1937-1948.

Høgsberg J and Krenk S (2015) Balanced calibration of resonant piezoelectric RL shunts with quasi-static background flexibility correction. Journal of Sound and Vibration 341:16-30.

Høgsberg J and Krenk S (2017) Calibration of piezoelectric RL shunts with explicit residual mode correction, Journal of Sound and Vibration 386:65-81

Hollkamp JJ (1994) Multimodal passive vibration suppression with piezoelectric materials and resonant shunts, Journal of Intelligent Material Systems and Structures, 5:49-57.

IEEE Inc. (1988) Standards on piezoelectricity ANS/IEEE Std 1761987, USA.

Krenk S (2005) Frequency analysis of the tuned mass damper. Journal of Applied Mechanics 72:936-942.

Krenk S and Høgsberg J (2014) Tuned mass absorber on a flexible structure. Journal of Sound and Vibration 333:1577-1595.

Krenk S and Høgsberg J (2016) Tuned resonant mass or inerterbased absorbers: Unified calibration with quasi-dynamic flexibility and inertia correction. Proceedings of the Royal Society A-Mathematical, Physical and Engineering Sciences 472:20150718 (23 pp).

Lossouarn B, Aucejo M, Deü J-F, Multon B (2017) Design of inductors with high inductance values for resonant piezoelectric damping. Sensors and Actuators A: Physical 259:68-76.

Park CH and Inman DJ (1999) A uniform model for series R-L and parallel R-L shunt circuits and power consumption. SPIE Proceedings 3668:797804.

Porfiri M, Maurini C, Pouget J. (2007) Identification of electromechanical modal parameters of linear piezoelectric structures, Smart Materials and Structures, 16:323-331.

Soltani, P, Kerschen G, Tondreau G, Deraemaeker A (2014) A Piezoelectric vibration damping using resonant shunt circuits: an exact solution. Smart Materials and Structures, 23:125014.

Thomas O, Deü J-F and Ducarne J (2009) Vibration of an elastic structure with shunted piezoelectric patches: efficient finite element formulation and electromechanical coupling coefficients. International Journal for Numerical Methods in Engineering 80:235-268.

Thomas O, Ducarne J, Deü J-F (2012) Performance of piezoelectric shunts for vibration reduction. Smart Materials and Structues 21:015008.

Trindade MA, Benjeddou A (2009) Effective electromechanical coupling coefficients of piezoelectric adaptive structures: critical evaluation and optimization. Mechanics of Advanced Materials and Structures 16:210-223.

Wu SY (1996) Piezoelectric shunts with a parallel R-L circuit for structural damping and vibration control. SPIE Proceedings 2720:259-269.

Wu SY (1998) Method for multiple mode piezoelectric shunting with single PZT transducer for vibration control. Journal of Intelligent Material Systems and Structures 12:991-998. 